\title{
Light Speed Expansion and Rotation of a Very Dark Machian Universe Having Internal Acceleration
}

\author{
U. V. S. Seshavatharam ${ }^{1}$, S. Lakshminarayana ${ }^{2}$ \\ ${ }^{1}$ Honorary Faculty, I-SERVE, Survey No-42, Hitech City, Hyderabad, India \\ ${ }^{2}$ Department of Nuclear Physics, Andhra University, Visakhapatnam, India \\ Email: seshavatharam.uvs@gmail.com,lnsrirama@gmail.com
}

How to cite this paper: Seshavatharam, U.V.S. and Lakshminarayana, S. (2020) Light Speed Expansion and Rotation of a Very Dark Machian Universe Having Internal Acceleration. International Journal of Astronomy and Astrophysics, 10, 247-283. https://doi.org/10.4236/ijaa.2020.103014

\section{Received: July 22, 2020}

Accepted: September 26, 2020

Published: September 29, 2020

Copyright $\odot 2020$ by author(s) and Scientific Research Publishing Inc. This work is licensed under the Creative Commons Attribution International License (CC BY 4.0).

http://creativecommons.org/licenses/by/4.0/

\begin{abstract}
We present a Machian model of Quantum Cosmology with full dark matter and light speed expansion and rotation. During galaxy formation and evolution, fraction of dark matter transforms to visible matter with a relation of the form, $\mathrm{m}_{-}$vis $=$constant ${ }^{*}\left(\mathrm{~m} \_ \text {dark }\right)^{2 / 3}$. Using this relation and replacing MOND's "critical acceleration" with "current cosmic maximum angular acceleration", galactic flat rotation speed range of (50 to 500) $\mathrm{km} / \mathrm{sec}$ can be fitted well. Estimated flat rotation speeds of DD168, Milky Way and UGC12591 are $49.96 \mathrm{~km} / \mathrm{sec}, 199.66 \mathrm{~km} / \mathrm{sec}$ and $521.75 \mathrm{~km} / \mathrm{sec}$ respectively. Based on these striking coincidences, it is possible to say that, MOND's approach is implicitly connected with cosmological estimation of $95 \%$ invisible matter. With reference to SPARC data for flat rotation speeds and current cosmic maximum angular acceleration, galactic total mass can be estimated. Considering galactic total mass, galactic visible mass and dark mass can be estimated. Proceeding further, galactic working radii, angular velocity and visible matter density can be estimated. Estimated Milky Way's effective radius is $293 \mathrm{kpc}$. Even though, this model is free from "big bang", "inflation", "dark energy", "flatness" and "red shift" issues, at $2.722 \mathrm{~K}$, estimated present Hubble parameter is $66.24 \mathrm{~km} / \mathrm{sec} / \mathrm{Mpc}$, cosmic radius is 146.3 times of the Hubble radius, angular velocity is 146.3 times lower than the Hubble parameter and cosmic age is 146.3 times of the Hubble age. With future observations and advanced telescopes, it may be possible to see far distant galaxies and very old stars far beyond the current observable cosmic radius.
\end{abstract}

\section{Keywords}

Planck Scale, Machian Universe, Speed of Light, Galactic Dark Matter, Galactic Visible Mass, Galactic Visible Mass Density, Cosmic Anisotropy, 
Galactic Internal Acceleration, Cosmic Graviton Wavelength

\section{Introduction}

We would like to emphasize the fact that, the basic principles of cosmology were developed when the subject of cosmology was in its budding stage. Friedmann made two simple assumptions about the universe [1] [2]. They can be stated in the following way.

1) When viewed at large enough scales, universe appears the same in every direction.

2) When viewed at large enough scales, universe appears the same from every location.

In this context, Hawking expressed that [2]: "There is no scientific evidence for the Friedmann's second assumption. We believe it only on the grounds of modesty: it would be the most remarkable if the universe looked the same in every direction around us, but not around other points in the universe".

In our earlier and recent published papers [3]-[20] we tried to highlight the basic drawbacks of big bang, inflation and galactic red shift in various possible ways. As of now, theoretically and observationally, with respect to inflation, isotropy, expansion speed, dark matter, dark energy and rotation, whole subject of cosmology is being driven into many controversies and dividing cosmologists into various groups with difference of opinions. On the other hand, very unfortunate thing is that, quantum cosmology point of view, "as a whole", progress is very poor [21]. Instead of discussing the controversies, we would like to propose a new model which can pave a new way for understanding and correlating astrophysical and cosmological observations in terms of quantum mechanics and general theory of relativity in a Machian view. It needs further study.

With reference to our very recent publication [20], in this version,

1) Implemented Mach's relation in place of Schwarzschild radius of black hole.

2) We tried to couple cosmic gravitational self energy density and cosmic thermal energy density.

3) Tried to define the Gamma parameter with the ratio of past and current temperatures [10] [11]. It helps in estimating the Hubble parameter with a direct relation rather than trial-error approach.

4) Made an attempt to bring clarity in the estimation of galactic dark matter.

5) Tried to define a dark matter reference mass unit of $3.523 \times 10^{38} \mathrm{~kg}$ in terms of current cosmic mass and Planck mass.

6) Tried to provide a clear procedure for understanding galactic dark matter via Inverse square law and cosmic angular acceleration.

7) Removed some topics pertaining to "Cold dark matter" and "Relativistic dark matter" of galaxies. 
8) Based on the available Spitzer Photometry and Accurate Rotation curves (SPARC) data, we have added Table 1 for the estimation of galactic total mass, visible mass, dark mass, working radii and visible matter density.

9) Considering low massive galaxies, we have added Table 2. By assuming the visible mass, its dark mass, total mass, flat rotation speed, working radii and visible mass density can be estimated.

With three simplified assumptions, an attempt has been made to develop a practical model of the universe. As so many galaxies are rotating and all the cosmic observations are being carried out with photons, we consider a light speed expanding [22] [23] [24] [25] [26] and light speed rotating universe. As galaxies are the key building blocks of the evolving universe and as all galaxies constitute massive rotating dark holes at their centers, we consider a growing and rotating Machian universe model [27]-[34]. That is why we call it a practical model. Interesting point to be noted is that, our model is absolutely free from "cosmic red shift" concept. Most important point to be noted is that, we have developed a very tight quantum gravity relation for correlating cosmic temperature and Hubble parameter independent of galactic red shifts and galactic distances. It can be applied to different time periods of the past.

\section{Holographic Principle and Mach's Relation}

According to G't Hooft, the combination of quantum mechanics and gravity requires the three dimensional world to be an image of data that can be stored on a two dimensional projection much like a holographic image [35] [36]. The "holographic principle" is a property of string theory and a supposed property of quantum gravity that states that the description of a volume of space can be thought of as encoded on a lower-dimensional boundary. Based on this concept, for the four dimensional spacetime universe, its three dimensional increasing volume can be set by Mach's principle, $\left(G M_{t} / c^{2} R_{t}\right) \cong 1$. Clearly speaking, information of the evolving universe can be extracted from $R_{t} \cong \frac{G M_{t}}{c^{2}}$. With this proposal, at any stage of cosmic evolution, a closed and massive universe can be defined with its minimum possible radius. One can find interesting technical discussion on this relation by D. W. Sciama, R. H. Dicke, C. Brans and G. J. Whitrow [27]-[34]. According to Lorenzo Barattini and Paolo Christillin, centrifugal force appears to be due to a relativistic effect of the counter-rotating Machian Universe [35].

\section{Reasons for Choosing Light Speed}

Based on the following reasons, we consider light speed as a special feature of cosmic expansion and rotation.

1) All cosmic observations are being studied with photons that propagate with "speed of light". Clearly speaking, since the beginning of modern cosmological observations, galactic receding speeds, galactic rotation curves, galactic visible 
mass and gravitational lensing are being studied with "light speed" photon as a key tool.

2) It is well believed that "gravity" propagates with light speed. Even though gravitational wave physics is having 100 years of theoretical history, in 2015 only scientists could able detect "gravitons" [36]. Very interesting and notable point is that gravitons also propagate with "speed of light". So far no ground based telescope or space telescope is able to catch gravitons for studying routine galactic properties. Frankly speaking, gravitational wave physics is in its budding stage for understanding and studying the routine galactic observations.

3) It is well established that electromagnetic interaction propagates with light speed.

4) It is well proved that, light speed is the ultimate speed of material particles.

5) So far, it has not yet been possible physically to measure the actual galactic receding speeds.

6) So far, it has not yet been possible to demonstrate and distinguish "space without matter" and "matter without space". In this ambiguous situation, without knowing the origins of "space" and "matter", it is quite illogical to say that, space drags massive galaxies at super luminal speeds.

7) So far, either at microscopic level or at macroscopic level, it has not yet been possible to establish a common understanding among quantum mechanics and gravity.

\section{Reasons for Considering Universe as a Machian Sphere}

Based on the following reasons, we consider a Machian universe.

1) Without a radial in-flow of matter in all directions towards one specific point, one cannot expect a big crunch and without a big crunch, one cannot expect a big bang. Really if there was a "big bang" in the past, with reference to formation of big bang as predicted by GTR and with reference to the cosmic rate of expansion that might have taken place simultaneously in all directions at a "naturally selected rate" about the point of big bang: "point" of big bang can be considered as the characteristic reference point of cosmic expansion in all directions. Thinking in this way, to some extent, point of big bang can be considered as a possible centre of cosmic evolution. If so, thinking about a centre less universe is illogical.

2) Mass and size pertaining to pre and post big bang [37] are unclear.

3) Whether Planck scale is associated with big bang or big bang is associated with Planck scale is also unclear.

4) As there exist no clear reasons for understanding the occurrence of exponential expansion, cosmologists are having different opinions on cosmic inflation [38].

5) So far, it has not yet been possible to establish solid connection between Planck scale and current physical parameters of the observable universe.

6) At any stage of cosmic expansion, if the universe wishes to maintain a 
closed boundary to have its size minimum, it is having an option to follow the "Mach's relation" at that time. It may be noted that, Machian radius is 2 times less than the Schwarzschild radius of a black hole. At any stage of cosmic evolution, if one is willing to consider the "Machian radius" of the evolving universe as its minimum possible radius, corresponding other characteristic cosmic physical parameters can be estimated/predicted easily and can be compared with time to time cosmological observations.

\section{List of Symbols}

At any stage of cosmic evolution,

1) Cosmic time $=t$

2) Cosmic Hubble parameter $=H_{t}$

3) Cosmic angular velocity $=\omega_{t}$

4) Ratio of Hubble parameter to angular velocity $=\Upsilon_{t}$

5) Cosmic radius $=R_{t}$

6) Cosmic total dark mass $=M_{t}$

7) Cosmic volume $=\Phi_{t}$

8) Cosmic temperature $=T_{t}$

9) Galactic distance from cosmic centre $=\left(r_{\text {Gdis }}\right)_{t}$

10) Galactic receding speed from cosmic centre $=\left(v_{\text {Gres }}\right)_{t}$

11) Increment in expansion distance $=\Delta\left(d_{\text {exp }}\right)_{t}$

12) Distance from cosmic centre $=\left(r_{d}\right)_{t}$

13) Wavelength of cosmic graviton $=\left(\lambda_{g w}\right)_{t}$

14) Frequency of cosmic graviton $=\left(v_{g w}\right)_{t}$

15) Galactic star rotation speed $=\left(V_{\text {Grot }}\right)_{t}$

16) Galactic angular velocity $=\left(\omega_{\text {Grot }}\right)_{t}$

17) Estimated visible mass of galaxy $=\left(M_{\text {Gvis }}\right)_{t}$

18) Estimated visible mass density of galaxy $=\left(\rho_{\text {Gvis }}\right)_{t}$

19) Estimated total mass of galaxy $=\left(M_{G \text { tot }}\right)_{t}$

20) Estimated dark mass of galaxy $=\left(M_{\text {Gdark }}\right)_{t}$

21) Ratio of dark mass of galaxy to visible mass of galaxy $=$ Dark mass factor $=$ $\left(X_{\text {Gdark }}\right)_{t}$

22) $\%$ Dark mass of galaxy $=\left(\frac{\left(X_{\text {Gdark }}\right)_{t}}{1+\left(X_{\text {Gdark }}\right)_{t}}\right) \times 100$

Note: Planck scale symbols can be understood with a subscript "pl" and current symbols can be understood with a subscript "0".

\section{Three Simple Assumptions}

We propose the following three assumptions.

Assumption-1: By following Mach's principle, right from the beginning of Planck scale, universe is expanding with speed of light and rotating with speed of light from and about the cosmic centre. It can be expressed with,

$$
G M_{t} \cong c^{2} R_{t}
$$




$$
\begin{aligned}
& R_{t} \cong \frac{G M_{t}}{c^{2}} \cong \frac{c}{\omega_{t}} \\
& \therefore M_{t} \cong \frac{c^{3}}{G \omega_{t}}
\end{aligned}
$$

Assumption-2: At any stage of cosmic evolution, ratio of Hubble parameter to angular velocity can be expressed as,

$$
\frac{H_{t}}{\omega_{t}} \cong\left\{1+\ln \left(\frac{\omega_{p l}}{\omega_{t}}\right)\right\} \cong\left\{1+\ln \left(\frac{T_{p l}}{T_{t}}\right)^{2}\right\} \cong \Upsilon_{t}
$$

where $\left(\omega_{p l}, T_{p l}\right) \cong$ Planck scale angular velocity and temperature respectively.

Assumption-3: Right from the beginning of Planck scale, at any stage of cosmic expansion, gravitational self energy density and thermal energy density are equal in magnitude and can be expressed as follows.

$$
\text { Let, Cosmic volume }=\Phi_{t} \cong\left(\frac{4 \pi}{3} R_{t}^{3}\right)
$$

Based on relations (3) and (5), gravitational self energy density can be expressed as,

$$
\begin{gathered}
\frac{\left(\rho_{g s c}\right)_{t} c^{2}}{\Phi_{t}} \cong-\left(\frac{3 G M_{t}^{2}}{5 R_{t}}\right) \div\left(\frac{4 \pi}{3} R_{t}^{3}\right) \cong-\frac{9 \omega_{t}^{2} c^{2}}{20 \pi G} \\
-\frac{9 \omega_{t}^{2} c^{2}}{20 \pi G}+a T_{t}^{4} \cong 0 \\
\frac{9 \omega_{t}^{2} c^{2}}{20 \pi G} \cong a T_{t}^{4}
\end{gathered}
$$

\section{Expressions for Cosmic Temperature and Age}

Rewriting relation (8) with respect to the radiation energy density constant, $a \cong \frac{\pi^{2} k_{B}^{4}}{15 \hbar^{3} c^{3}}$ and considering relation (3), cosmic temperature can be estimated in the following way.

Based on assumption (3) and relation (8),

$$
\frac{\pi^{2} k_{B}^{4} T_{t}^{4}}{15 \hbar^{3} c^{3}} \cong \frac{9 \omega_{t}^{2} c^{2}}{20 \pi G}
$$

By proceeding in the following way, a very simple expression for cosmic temperature can be obtained.

$$
\begin{aligned}
k_{B}^{4} T_{t}^{4} & \cong\left(\frac{135}{20 \pi^{3}}\right) \frac{\omega_{t}^{2} \hbar^{3} c^{5}}{G} \\
& \cong\left(\frac{135}{20 \pi^{3}}\right) \frac{\omega_{t}^{2} \hbar^{3} c^{5}}{G} \times\left(\frac{G \hbar c}{G \hbar c}\right) \\
& \cong\left(\frac{135}{20 \pi^{3}}\right) \frac{\omega_{t}^{2} \hbar^{4} c^{6}}{G^{2}}\left(\frac{\hbar c}{G}\right)^{-1}
\end{aligned}
$$




$$
\begin{aligned}
& \cong\left(\frac{135}{20 \pi^{3}}\right) \frac{\omega_{t}^{2} \hbar^{4} c^{6}}{G^{2}}\left(\sqrt{\frac{\hbar c}{G}}\right)^{-2} \times\left(\frac{4 G^{2} c^{6}}{4 G^{2} c^{6}}\right) \\
& \cong\left(\frac{135}{20 \pi^{3}}\right) \frac{\hbar^{4} c^{12}}{G^{4}}\left(\sqrt{\frac{\hbar c}{G}}\right)^{-2} \times\left(\frac{c^{3}}{2 G \omega_{t}}\right)^{-2} \\
& \cong\left(\frac{135}{20 \pi^{3}}\right) \frac{\hbar^{4} c^{12}}{G^{4} M_{p l}^{2} M_{t}^{2}}
\end{aligned}
$$

Hence,

$$
T_{t} \cong\left(\frac{135}{20 \pi^{3}}\right)^{\frac{1}{4}} \frac{\hbar c^{3}}{k_{B} G \sqrt{M_{t} M_{p l}}} \cong \frac{0.6831 \hbar c^{3}}{k_{B} G \sqrt{M_{t} M_{p l}}}
$$

It may be noted that, in a theoretical approach,

1) Right from the beginning of Planck's scale, we try to see the gradual time dependent variations in cosmic radius, cosmic temperature, Hubble parameter and angular velocity with a set of three simple assumptions. With further study, deviations in their respective magnitudes can be analyzed with reference to radiation dominated era, matter dominated era like physical conditions and black body spectrum like analyzing tools and methods. We are working in this direction.

2) As cosmic mass, radius, Hubble parameter, angular velocity and temperature are inter-related, they can be represented in any required manner.

3) "Ease" and "Simplicity" are the basic requirements of unification.

As universe is always expanding with speed of light, $R_{t} \cong c t$. Hence,

$$
t \cong \frac{R_{t}}{c} \cong \frac{1}{\omega_{t}} \cong \frac{\Upsilon_{t}}{H_{t}}
$$

\section{Current Cosmic Physical Parameters at Current Cosmic Temperature}

Following the assumptions, the Planck scale Hubble parameter can be expressed as follows:

$$
\omega_{p l} \cong \frac{c^{3}}{G M_{p l}} \cong \frac{c}{R_{p l}} \cong H_{p l} \cong 1.85492 \times 10^{43} \mathrm{sec}^{-1}
$$

where $R_{p l} \cong G M_{p l} / c^{2} \cong \sqrt{G \hbar / c^{3}} \cong 1.6162 \times 10^{-35} \mathrm{~m}$ is the Planck length and the assumed radius connected with the Planck mass.

Planck scale cosmic temperature can be expressed as

$$
T_{p l} \cong\left(\frac{9 H_{p l}^{2} c^{2}}{20 \pi G a}\right)^{1 / 4} \cong\left(\frac{9 \omega_{p l}^{2} c^{2}}{20 \pi G a}\right)^{1 / 4} \cong 9.67791 \times 10^{31} \mathrm{~K}
$$

As per the 2015 Planck data (Planck Collaboration 2015) [39],

The current value of the Hubble parameter is reported to be:

$$
\left.\begin{array}{l}
\text { Planck TT + low P : }(67.31 \pm 0.96) \mathrm{km} / \mathrm{sec} / \mathrm{Mpc} \\
\text { Planck TE + low P : }(67.73 \pm 0.92) \mathrm{km} / \mathrm{sec} / \mathrm{Mpc} \\
\text { Planck TT, TE, EE + low P : }(67.7 \pm 0.66) \mathrm{km} / \mathrm{sec} / \mathrm{Mpc}
\end{array}\right\}
$$


The current value of CMBR temperature is reported to be:

$$
\left.\begin{array}{l}
\text { Planck TT + low P + BAO : }(2.722 \pm 0.027) \mathrm{K} \\
\text { Planck TT; TE; EE + low P + BAO : }(2.718 \pm 0.021) \mathrm{K}
\end{array}\right\}
$$

In this paper, for calculation purpose, we consider $T_{0} \cong 2.722 \mathrm{~K}$. Hence,

$$
\begin{gathered}
\Upsilon_{0} \cong\left\{1+\ln \left(\frac{T_{p l}}{T_{0}}\right)^{2}\right\} \cong 146.3 \\
\omega_{0} \cong \sqrt{\frac{20 \pi G a T_{t}^{4}}{9 c^{2}} \cong 1.4674 \times 10^{-20} \mathrm{rad} \cdot \mathrm{sec}^{-1}} \\
H_{0} \cong \Upsilon_{0} \omega_{0} \cong 2.14675 \times 10^{-18} \mathrm{sec}^{-1} \cong 66.24 \mathrm{~km} / \mathrm{sec} / \mathrm{Mpc} \\
R_{0} \cong \frac{c}{\omega_{0}} \cong \Upsilon_{0}\left(\frac{c}{H_{0}}\right) \cong 2.043 \times 10^{28} \mathrm{~m} \\
M_{0} \cong \frac{c^{3}}{G \omega_{0}} \cong 2.751 \times 10^{55} \mathrm{~kg} \\
t_{0} \cong \frac{1}{\omega_{0}} \cong \frac{\Upsilon_{0}}{H_{0}} \cong 2159.5 \text { Billion years }
\end{gathered}
$$

\section{Cosmic Rotation}

As per the recent 2020 publication [40], according to Vladimir A. Korotky, Eduard Masár and Yuri N. Obukhov: "In observational cosmology, the main difficulty for detecting a global rotation is its smallness-less than $10^{-13} \mathrm{rad} / \mathrm{year}$ according to the generally accepted assessment. It is impossible in the Universe to distinguish the direction corresponding to the axis of rotation, with respect to which one could notice deviations (in the standard tests) from the Friedman standard cosmology. In theoretical cosmology, the main difficulties are related, on the one hand, to the lack of simple models of an expanding and rotating Universe in general relativity (GR) similar to Friedman-Robertson-Walker models. On the other hand, there are no convincing predictive effects of cosmic rotation that are consistent with the capabilities of the equipment of modern astronomical observatories".

In this context, we strongly propose that, as "spin" is a basic property of quantum mechanics, from the subject point of quantum gravity, universe must have "rotation". If it is assumed that, universe is a Machian sphere, it is quite natural to expect "cosmic rotation". Considering the 6 major applications of dark matter in view (see section 10), by estimating the currently believed dark mass of a galaxy with its corresponding visible mass, and by replacing the "acceleration" parameter $\left[\approx\left(\mathrm{cH}_{0}\right) / 6\right]$ of MOND with cosmic angular acceleration $\left(c \omega_{0}\right)$, we try to fit the flat rotation speed of a galaxy. One most interesting as well as speculative point is that, even though MOND approach is "the best" in fitting galactic rotation curves, its back ground physics is unclear with respect to galactic structures and cosmic acceleration parameter $\left(\mathrm{cH}_{0}\right)$. It can be con- 
firmed with the conclusion section of recent paper authored by Stacy McGaugh [41]. Here, our approach is having 4 different actions:

1) To estimate the generally believed dark mass of galaxy.

2) To implement cosmic angular acceleration in understanding galactic structure.

3) To fit flat rotation speed of a galaxy.

4) Finally, to divulge the existence of cosmic rotation.

Kurt Gödel put a lot of efforts in developing a realistic universe model with rotation and expansion. His heuristic results were presented at International Congress of Mathematics held at Cambridge (Massachusetts) from 30th August to 5th September 1950 [42].

The first experimental evidence of the Universe rotation was done by Birch in 1982 evidently [43]. According to Birch, there appears to be strong evidence that the Universe is anisotropic on a large scale, producing position angle offsets in the polarization and brightness distributions of radio sources. These can probably be explained on the basis of a rotation of the Universe with an angular velocity of approximately $10^{-13} \mathrm{rad} /$ year. In our model, current cosmic angular velocity is $4.631 \times 10^{-13} \mathrm{rad} /$ year. Observational effects of current cosmic rotation can be understood with the works of Obukhov [44], Godlowski [45], Longo [46] and Chechin [47].

According to Michael Longo the universe has a net angular momentum and was born in a spin.

Whittaker says [48]: "however, that any of the mathematical-physical theories that have been put forward to explain spin (rotation) in the universe has yet won complete and universal acceptance; but progress has been so rapid in recent years that it is reasonable to hope for a not long-delayed solution of this fundamental problem of cosmology".

According to T. Valery and S. V. Timkov, current universe is rotating with light speed and angular velocity equal to the current Hubble parameter [49].

Very recent and advanced studies of Lior Shamir suggest [50] that, the distribution of galaxy spin directions in SDSS and Pan-STARRS shows patterns in the asymmetry between galaxies with opposite spin directions and can be considered as an evidence for large-scale anisotropy and an indication for a rotating universe.

\section{Understanding Galactic Dark Matter and Its Estimation}

As per modern cosmological observations, most of the cosmologists infer dark matter as a characteristic and inherent feature of any galaxy. Dark matter seems to have a major role in understanding 6 different issues pertaining to many of the galaxies. They are [51] [52]:

1) Galactic formation and evolution.

2) Galactic rotational curves.

3) Gravitational lensing. 
4) Galactic collisions.

5) Motion of galaxies within galaxy clusters.

6) Cosmic microwave fluctuations.

Most unfortunate thing is that, so far, no ground based experiment or no cosmological observation could establish any direct evidence for the existence of dark matter and opened a new window for MOND like interesting concepts. In this context, some of the cosmologists are trying to understand the presumed 6 major applications of dark matter with galactic "visible mass" only. Effectiveness of this attempt seems to be interesting. One can find interesting technical discussion in the context of CMB fluctuations, galactic rotation curves and galaxy clusters [53]. Anyhow, here we would like appeal that, the subject under consideration is falling under a "debate" or "conflict of interest" and needs further study at utmost fundamental level with respect to the strange nature of presumed dark matter. Ongoing and future experiments and observations may help in resolving the issue.

In this context we propose our views in the following way.

1) Right from the beginning of Planck scale, "universe" is generating dark mass only.

2) Dark mass plays the complete role in the formation and evolution of galaxies.

3) During cosmic evolution, for any galaxy, part of dark mass slowly transforms to visible mass.

4) Current galactic visible mass can be understood with the following relation.

$$
\left(M_{\text {Gvis }}\right)_{0} \cong\left[\left(M_{X}\right)_{0}\left(M_{\text {Gdark }}\right)_{0}^{2}\right]^{\frac{1}{3}}
$$

where $\left(M_{\text {Gvis }}\right)_{0}=$ Current visible mass of galaxy.

$$
\left(M_{\text {Gdark }}\right)_{0}=\text { Dark mass of galaxy. }
$$

$$
\left(M_{X}\right)_{0} \cong \frac{\left(M_{0}^{3} M_{p l}\right)^{\frac{1}{4}}}{\left(1+\sqrt{\Upsilon_{0}}\right)} \cong 3.523 \times 10^{38} \mathrm{~kg}
$$

$=$ Current semi empirical mass of reference [20].

5) By estimating the current galactic visible mass, current galactic dark mass can be understood with the following relations.

$$
\begin{gathered}
\left(M_{\text {Gdark }}\right)_{0} \cong \frac{\sqrt[3]{\left(M_{\text {Gvis }}\right)_{0}}}{\sqrt{\left(M_{X}\right)_{0}}} \\
\left(M_{\text {Gdark }}\right)_{0} \propto\left(M_{\text {Gvis }}\right)_{0}^{3 / 2}
\end{gathered}
$$

6) Current galactic dark mass factor can be understood with the following relation.

$$
\left(X_{\text {Gdark }}\right)_{0} \cong \frac{\left(M_{\text {Gdark }}\right)_{0}}{\left(M_{\text {Gvis }}\right)_{0}} \cong \sqrt{\frac{\left(M_{\text {Gvis }}\right)_{0}}{\left(M_{X}\right)_{0}}}
$$


where, $\left(X_{\text {Gdark }}\right)_{0}=$ Current dark mass factor of galaxy.

$$
\left(M_{\text {Gdark }}\right)_{0} \cong\left(X_{\text {Gdark }}\right)_{0}\left(M_{\text {Gvis }}\right)_{0}
$$

7) Current galactic total mass can be understood with the following relation.

$$
\left(M_{\text {Gtot }}\right)_{0} \cong\left(M_{\text {Gdark }}\right)_{0}+\left(M_{\text {Gvis }}\right)_{0} \cong\left[1+\left(X_{\text {Gdark }}\right)_{0}\right]\left(M_{\text {Gvis }}\right)_{0}
$$

where, $\left(M_{G \text { tot }}\right)_{0}=$ Current total mass of galaxy.

8) Current galactic dark mass percentage can be understood with the following relation.

$$
\%\left(M_{\text {Gdark }}\right)_{0} \cong \frac{\left(M_{\text {Gdark }}\right)_{0}}{\left(M_{\text {Gtot }}\right)_{0}} \times 100 \cong \frac{\left(X_{\text {Gdark }}\right)_{0}}{\left[1+\left(X_{\text {Gdark }}\right)_{0}\right]} \times 100
$$

\section{To Develop a MOND like Relation for Galactic Flat Orbiting Speed with Cosmic Angular Velocity and Galactic Total Mass}

Even though MOND approach [54] [55] [56] was aimed for understanding galactic rotation curves without dark matter, with reference to the proposed current cosmic angular velocity and relation (10), it is possible to fit the rotation curves and thereby galactic dark masses can be inferred.

Observed galactic flat rotation curves can be understood in the following way. At present, for any galaxy, let,

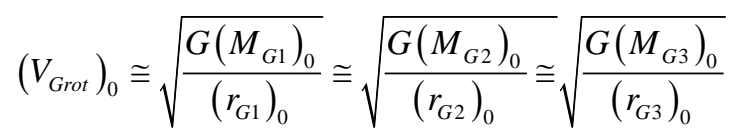

where,

$\left(V_{\text {Grot }}\right)_{0}=$ Current observed flat orbiting velocity of galactic star.

$\left(r_{G 1}\right)_{0},\left(r_{G 2}\right)_{0},\left(r_{G 3}\right)_{0} \cong$ Increasing galactic distances from galactic centre.

$\left(M_{G 1}\right)_{0},\left(M_{G 2}\right)_{0},\left(M_{G 3}\right)_{0} \cong$ Increasing galactic masses at increasing galactic radii.

$$
\left(\frac{\left(M_{G 1}\right)_{0}}{\left(r_{G 1}\right)_{0}} \cong \frac{\left(M_{G 2}\right)_{0}}{\left(r_{G 2}\right)_{0}} \cong \frac{\left(M_{G 3}\right)_{0}}{\left(r_{G 3}\right)_{0}} \cong \text { Constant }\right)
$$

Point to be noted is that, star's orbiting velocity changes with changing galactic dark mass distribution and it needs further study and observational data for a number of galaxies. Let,

$$
\left(V_{\text {Grot }}\right)_{0} \cong \sqrt{\frac{G\left(M_{\text {Gtot }}\right)_{0}}{\left(r_{\text {Geffe }}\right)_{0}}}
$$

where,

$\left(V_{\text {Grot }}\right)_{0} \cong$ Observed current flat orbiting speed of galactic star.

$\left(r_{\text {Geffe }}\right)_{0} \cong$ Current galactic effective radius.

Writing $\left(r_{\text {Geffe }}\right)_{0} \cong \frac{G\left(M_{\text {Gtot }}\right)_{0}}{\left(V_{\text {Grot }}\right)_{0}^{2}}$ and eliminating $\left(r_{\text {Geffe }}\right)_{0}$, 


$$
\frac{G\left(M_{\text {Gtot }}\right)_{0}}{\left(r_{\text {Geffe }}\right)_{0}^{2}} \cong \frac{\left(v_{\text {Grot }}\right)_{0}^{4}}{G\left(M_{\text {Gtot }}\right)_{0}}
$$

Now, based on MOND approach, assume that,

$$
\frac{\left(v_{\text {Grot }}\right)_{0}^{4}}{G\left(M_{\text {Gtot }}\right)_{0}} \cong c \omega_{0}
$$

where,

$c \omega_{0} \cong$ Current possible maximum cosmic angular acceleration.

Thus,

$$
\left(V_{\text {Grot }}\right)_{0} \cong \sqrt[4]{G\left(M_{G \text { tot }}\right)_{0} c \omega_{0}} \cong \sqrt[4]{G\left[1+\left(X_{\text {Gdark }}\right)_{0}\right]\left(M_{G v i s}\right)_{0} c \omega_{0}}
$$

Based on this relation, estimated flat rotation speeds of DD168, Milky Way and UGC12591 are $49.96 \mathrm{~km} / \mathrm{sec}, 199.66 \mathrm{~km} / \mathrm{sec}$ and $521.75 \mathrm{~km} / \mathrm{sec}$ respectively. As per the reference photometric data [54] and [57], their corresponding flat rotation speeds are $52 \mathrm{~km} / \mathrm{sec}, 202.6 \mathrm{~km} / \mathrm{sec}$ and $500 \mathrm{~km} / \mathrm{sec}$ respectively. Within a range of (50 to 500) $\mathrm{km} / \mathrm{sec}$, these striking coincidences are strongly supporting our proposed concepts.

In this way,

1) Current galactic angular velocity can be understood with the following relations.

$$
\left(\omega_{\text {Grot }}\right)_{0} \cong \frac{\left(V_{\text {Grot }}\right)_{0}^{3}}{G\left(M_{\text {Gtot }}\right)_{0}}
$$

where, $\left(\omega_{\text {Grot }}\right)_{0}=$ Current galactic angular velocity

$$
\left(V_{\text {Grot }}\right)_{0}\left(\omega_{\text {Grot }}\right)_{0} \cong c \omega_{0}
$$

2) Based on relations (28), (31) and (32), effective radius of galaxy can be expressed as,

$$
\left(r_{\text {Geffe }}\right)_{0} \cong \frac{G\left(M_{\text {Gtot }}\right)_{0}}{\sqrt{G\left(M_{\text {Gtot }}\right)_{0} c \omega_{0}}} \cong \sqrt{\frac{G\left(M_{\text {Gtot }}\right)_{0}}{c \omega_{0}}}
$$

3) Based on relation (28), as a special case, radius of galaxy corresponding to its visible mass and flat rotation speed, can be called as galactic "visible radius" and can be expressed as,

$$
\left(r_{\text {Gvis }}\right)_{0} \cong \frac{G\left(M_{\text {Gvis }}\right)_{0}}{\sqrt{G\left(M_{\text {Gtot }}\right)_{0} c \omega_{0}}} \cong \sqrt{\frac{\left(M_{\text {Gvis }}\right)_{0}}{\left[1+\left(X_{\text {Gdark }}\right)_{0}\right] c \omega_{0}}}
$$

4) Based on relations (28), (34) and (35), if dark matter distribution is "as expected", galaxy should follow flat rotation speeds in between $\left(r_{\text {Gvis }}\right)_{0}$ and $\left(r_{\text {Geffe }}\right)_{0}$. A least, close to the geometric mean of $\left(r_{\text {Gvis }}\right)_{0}$ and $\left(r_{\text {Geffe }}\right)_{0}$ rotation speed should be flat. It can be expressed as,

$$
\left(r_{\text {Ggeom }}\right)_{0} \cong \sqrt{\left(r_{\text {Geffe }}\right)_{0}\left(r_{\text {Gvis }}\right)_{0}} \cong \sqrt{\frac{G\left(M_{\text {Gvis }}\right)_{0}}{c \omega_{0}}}
$$


5) Effective, geometric and visible radii can be expressed with a common relation of the form,

$$
\begin{aligned}
& \left(r_{G x}\right)_{0} \cong\left[1+\left(X_{G d a r k}\right)_{0}\right]^{p} \sqrt{\frac{G\left(M_{G v i s}\right)_{0}}{c \omega_{0}}} \\
& \text { where, } p=\left(+\frac{1}{2}, 0,-\frac{1}{2}\right)
\end{aligned}
$$

6) With reference to the observed ordinary matter density [39] [58], we noticed that, for any galaxy,

$$
\left(\rho_{\text {Gvis }}\right)_{0} \approx\left[\frac{\left(V_{\text {Grot }}\right)_{0}}{c}\right]\left(\frac{\sqrt{\left(M_{\text {Gvis }}\right)_{0}\left(M_{\text {Gtot }}\right)_{0}}}{\frac{4 \pi}{3}\left(r_{\text {Geffe }}\right)_{0}^{3}}\right) \approx(1.0 \text { to } 29.2) \times 10^{-28} \mathrm{~kg} \cdot \mathrm{m}^{-3}
$$

Clearly speaking, fitted density seems to be the geometric mean of visible mass density and total mass density. For 122 galaxies, its average value is close to $6.5 \times$ $10^{-28} \mathrm{~kg} / \mathrm{m}^{3}$. It needs further study at basic level. See Table 1 and its last column. Interesting point to be noted is that, $\left[\frac{\left(V_{G r o t}\right)_{0}}{c}\right]$ seems to play a key role in fitting the mean density. It needs further study.

It may also be noted that, based on the average mass-to-light ratio for any galaxy, present matter density can be expressed with the following relation [58].

$$
\text { Here, }\left\{\begin{array}{l}
\quad\left(\rho_{\text {Gvis }}\right)_{0} \cong 1.5 \times 10^{-32} \eta h_{0} \text { gram } / \mathrm{cm}^{3} \\
h_{0} \cong H_{0} / 100 \mathrm{~km} / \mathrm{sec} / \mathrm{Mpc} \cong 0.68
\end{array}\right.
$$

Note that elliptical galaxies probably comprise about $60 \%$ of the galaxies in the universe and spiral galaxies thought to make up about $20 \%$ percent of the galaxies in the universe. Almost $80 \%$ of the galaxies are in the form of elliptical and spiral galaxies. For spiral galaxies, $\eta h_{0}^{-1} \cong 9 \pm 1$ and for elliptical galaxies, $\eta h_{0}^{-1} \cong 10 \pm 2$. For our galaxy inner part, $\eta h_{0}^{-1} \cong 6 \pm 2$. Thus the average $\eta h_{0}^{-1}$ is very close to 8 to 9 and its corresponding matter density is close to (5.55 to $6.24) \times 10^{-32} \mathrm{gram} / \mathrm{cm}^{3}$ and can be compared with the above proposed magnitude of (1.0 to 29.2$) \times 10^{-28} \mathrm{~kg} \cdot \mathrm{m}^{-3}$.

Note: Important point to be noted is that, in estimating the approximate visible mass of the universe, while multiplying the average galactic visible matter density with total cosmic volume, one must exclude the intergalactic volume or volume occupied by the free space. Clearly speaking, one must find the ratio of total galactic volume and total free space volume.

7) With reference to MSTG and MOND approaches [54], approximate galactic core radius can be expressed as,

$$
\left(r_{\text {Gcore }}\right)_{0} \approx \frac{\left(r_{\text {Gvis }}\right)_{0}}{2 \pi} \approx\left(\frac{1}{2 \pi}\right) \sqrt{\frac{G\left(M_{\text {Gvis }}\right)_{0}}{\left(X_{\text {dark }}+1\right) c \omega_{0}}}
$$




\section{To Understand Cosmic Redshift Associated with Cosmic Current and Past Temperatures}

With reference to the light emitted from first hydrogen atoms, we define the following two ad hoc relations.

$$
\text { Let, } \quad x_{t} \cong 1+\ln \left(\frac{R_{t}}{R_{p l}}\right)
$$

where $R_{p l}$ is the Planck scale cosmic radius.

$$
Z \cong \sqrt{\exp \left(x_{0}-x_{t}\right)}-1
$$

Based on these two ad hoc definitions, it is possible to show that,

$$
\begin{aligned}
Z & \cong \sqrt{\exp \left\{\left[1+\ln \left(\frac{R_{0}}{R_{p l}}\right)\right]-\left[1+\ln \left(\frac{R_{t}}{R_{p l}}\right)\right]\right\}}-1 \\
& \cong \sqrt{\exp \left\{\left(\ln \left(\frac{R_{0}}{R_{p l}}\right)-\ln \left(\frac{R_{t}}{R_{p l}}\right)\right)\right\}}-1 \\
& \cong \sqrt{\exp \left\{\ln \left(\frac{R_{0}}{R_{t}}\right)\right\}}-1 \cong \sqrt{\frac{R_{0}}{R_{t}}}-1
\end{aligned}
$$

With respect to the proposed assumptions, it is clear that at any stage of cosmic expansion,

1) Cosmic radius is inversely proportional to cosmic angular velocity.

2) Cosmic angular velocity is directly proportional to squared cosmic temperature.

Hence,

$$
Z \cong \sqrt{\frac{R_{0}}{R_{t}}}-1 \cong \sqrt{\frac{\omega_{t}}{\omega_{0}}}-1 \cong \sqrt{\frac{T_{t}^{2}}{T_{0}^{2}}}-1 \cong \frac{T_{t}}{T_{0}}-1
$$

where $T_{t}$ is the past cosmic temperature; and $T_{0}$ is the current cosmic temperature.

$$
\therefore Z+1 \cong \frac{T_{t}}{T_{0}}
$$

\section{To Understand Hubble's Law and to Locate the Cosmic Centre}

Based on first assumption and special theory of relativity, from and about the cosmic centre, for any materialistic galaxy, its current receding speed can be understood in the following way.

$$
\left(v_{\text {Gres }}\right)_{0} \cong\left(\frac{\left(r_{\text {Gdis }}\right)_{0}}{R_{0}}\right) c \cong\left(r_{\text {Gdis }}\right)_{0} \omega_{0} \cong\left(\frac{1}{\Upsilon_{0}}\right)\left(r_{\text {Gdis }}\right)_{0} H_{0}
$$

In this way qualitatively Hubble's [59] law can be understood. Since $\omega_{0}$ is known, by knowing the actual galactic receding speed, its distance from the 
cosmic centre can be estimated. By estimating the cosmic radial distances of galaxies along with their locations, it seems possible to locate the cosmic centre. If any galaxy's actual receding speed is found to be faster than speed of light, our model can be falsified.

\section{To Estimate Current Gravitational Wave Length}

With reference to current cosmic mass and Planck mass, wavelength of current gravitational waves [60] can be obtained as follows.

Our idea is that, at any stage of cosmic evolution, "evolving universe" is an "internal accelerating" object and wavelength of cosmic graviton is equal to $2 \pi$ times the geometric mean of radius of universe at time $t$ and Planck scale radius. At present, it can be expressed as,

$$
\left(\lambda_{g w}\right)_{0} \cong 2 \pi \sqrt{R_{0} R_{p l}} \cong \frac{2 \pi c}{\sqrt{\omega_{0} \omega_{p l}}} \cong \frac{2 \pi G \sqrt{M_{0} M_{p l}}}{c^{2}} \cong 0.00361 \mathrm{~m}
$$

Corresponding frequency and energy can be expressed as,

$$
\begin{aligned}
& \left(v_{g w}\right)_{0} \cong \frac{c}{\left(\lambda_{g w}\right)_{0}} \cong \frac{c}{2 \pi \sqrt{R_{0} R_{p l}}} \cong \frac{\sqrt{\omega_{0} \omega_{p l}}}{2 \pi} \cong \frac{c^{3}}{2 \pi G \sqrt{M_{0} M_{p l}}} \cong 83.033 \mathrm{GHz} \\
& \left(E_{g w}\right)_{0} \cong h\left(\frac{\sqrt{\omega_{0} \omega_{p l}}}{2 \pi}\right) \cong \hbar \sqrt{\omega_{0} \omega_{p l}} \cong 0.0003434 \mathrm{eV}
\end{aligned}
$$

In a rotating universe, according to Christillin [61], there is a possibility of continuous generation of gravitational waves. In this context, our proposed $\frac{\sqrt{\omega_{0} \omega_{p l}}}{2 \pi} \cong 83 \mathrm{GHz}$ cosmic gravitons are for future experimental verification.

\section{Understanding Cosmic Anisotropy}

As universe is always expanding at speed of light, at any stage of expansion, cosmic boundary expands by $3 \times 10^{8} \mathrm{~m}$ in one second. In between the cosmic centre and cosmic boundary, expansion distance covered in one second can be expressed as,

$$
\Delta\left(d_{\text {exp }}\right)_{t} \cong \frac{\left(r_{d}\right)_{t}}{R_{t}}\left(3 \times 10^{8}\right) \mathrm{m}
$$

where,

$$
\begin{aligned}
& \Delta\left(d_{\text {exp }}\right)_{t}=\text { Increment in expansion distance at time } t . \\
& \left(r_{d}\right)_{t}=\text { Distance from cosmic centre at time } t . \\
& R_{t}=\text { Cosmic radius at time } t .
\end{aligned}
$$

Clearly speaking,

1) Distance moved near to cosmic boundary is more compared to distance moved near to cosmic centre.

2) Rate of volume change near to cosmic boundary is higher than the rate of 
volume change near to cosmic centre.

3) Anisotropy [50] [62] gradually increases from cosmic centre to cosmic boundary.

\section{Understanding (Internal) Cosmic Acceleration}

According to Saul Perlmutter, Adam Riess and Brian Schmidt, observable universe is accelerating [63] [64]. Clearly speaking, expansion of the universe is such that the velocity at which a distant galaxy is receding from the observer is continuously increasing with time. It can be understood in the following way.

Based on relation (47) and with reference to two time periods $\left(t_{2}>t_{1}\right)$, ratio of galactic receding speeds can be expressed as,

$$
\frac{\left(v_{\text {Gres }}\right)_{t_{2}}}{\left(v_{\text {Gres }}\right)_{t_{1}}} \cong\left(\frac{\left(r_{\text {Gdis }}\right)_{t_{2}}}{\left(r_{\text {Gdis }}\right)_{t_{1}}}\right)\left(\frac{R_{t_{1}}}{R_{t_{2}}}\right)
$$

where,

$\left(v_{\text {Gres }}\right)_{t_{1}}$ and $\left(v_{\text {Gres }}\right)_{t_{2}}=$ Galactic receding speeds corresponding to $t_{1}, t_{2}$ respectively where $\left(v_{\text {Gres }}\right)_{t_{2}}>\left(v_{\text {Gres }}\right)_{t_{1}}$.

$\left(r_{\text {Gdis }}\right)_{t_{1}}$ and $\left(r_{\text {Gdis }}\right)_{t_{2}}=$ Galactic distances corresponding to $t_{1}, t_{2}$ respectively where $\left(r_{\text {Gdis }}\right)_{t_{2}}>\left(r_{\text {Gdis }}\right)_{t_{1}}$.

$R_{t_{1}}$ and $R_{t_{2}}=$ Theoretical cosmic radii corresponding to $t_{1}, t_{2}$ respectively where $\left(R_{t_{2}}>R_{t_{1}}\right)$.

Clearly speaking,

1) Within the cosmic horizon, second by second, galactic receding speeds are increasing and resemble a kind of internal cosmic acceleration.

2) Acceleration seems to be higher near to cosmic centre and gradually reaches to zero at horizon.

3) Hubble's law pertaining to two increasing time periods seems to be a natural consequence of internal cosmic acceleration.

4) Cosmic horizon is always expanding at speed of light.

\section{To Relinquish Dark Energy}

If it is assumed that, universe is always expanding with speed of light, then, considering "dark energy" like concepts need not be required [65] [66]. If one is able to understand the reasons for light speed expansion, it may help in understanding the internal acceleration. Proceeding further, till today, no cosmological observation or no ground based experiment could shed light on the physical nature of dark energy.

\section{Understanding Cosmic Age}

Observable cosmic radius is just 2.2 times the Hubble radius and corresponding cosmic age is $\left(1 / H_{0}\right)$. Our model result of cosmic radius is 146.3 times the Hubble radius and corresponding light speed cosmic age is 146.3 times $\left(1 / H_{0}\right)$. In this way, our model result of cosmic age can be justified. We would like to 
emphasize that,

1) Modern cosmological observations are limited to 2.2 times the Hubble radius and needs further study.

2) Time is a dynamic and emerging cosmic parameter.

3) Cosmic age depends on the model under consideration.

4) One should not worry about the absolute value of cosmic age.

\section{Understanding Nucleosynthesis}

Based on relations (1) to (20) and by assuming appropriate density range or temperature range that is required for formation of nucleons and atoms, cosmic physical parameters pertaining to nucleosynthesis can be understood [67]. For example, cosmic age corresponding to a temperature of $10^{10} \mathrm{~K}$ is $5.05 \mathrm{sec}$. It needs further study. Estimated cosmic age corresponding to $3000 \mathrm{~K}$ is $1.78 \times 10^{6}$ years. This estimation is 4.68 times higher than the current estimation of $3.8 \times$ $10^{5}$ years. Clearly speaking, starting from the Planck scale, without considering "inflation" like cooling pattern, our model follows a slow thermal cooling pattern throughout the cosmic evolution.

\section{Inferences of Growing Machian Universe}

Based on the proposed assumptions and with reference to the above relations, we would like to say that,

1) Earth, Solar family, Milky Way and all other galaxies are living inside the proposed Machian universe.

2) There is matter and space outside the proposed growing and rotating Machian universe.

3) The growing and rotating Machian universe always sucks matter inward and thus it grows on with increasing suction rate.

4) At poles, inward matter flow rate is maximum and at equator, inward matter flow rate is zero. Thus, starting from poles to equator, inward matter flow rate gradually decreases.

\section{To Develop Practical Methods for Understanding a Growing Machian Universe}

We would like to propose the following points for understanding cosmic singularity.

1) To study cosmic anisotropy on very large cosmic distances.

2) To believe, to understand and to study the consequences of cosmic rotation.

3) To develop high precision cosmic gyroscopes.

4) To study galactic mean temperature and to estimate the galactic dark mass.

5) To correlate galactic rotations and cosmic rotation.

6) To find oldest galaxies like EGSY8p7 whose age is closer to or greater than 13.8 billion years. 
7) To study very high energy cosmic gravitons.

8) To study cosmic dipole magnetic moment and its related properties.

\section{Discussion on Cosmic Temperature, Angular Velocity, Radius and Age}

Relation (11) is very similar to the famous Hawking's black hole temperature formula [68]. This is due to the similarities in between Mach's relation for increasing cosmic radii and Schwarzschild radius of a black hole. For the Planck scale, cosmic temperature can be expressed as,

$$
T_{p l} \cong \frac{0.6831 \hbar c^{3}}{k_{B} G M_{p l}}
$$

Comparing this with Hawking's relation, it is differing by the numerical factor 0.6831. In may be noted that, in our earlier publications [3] [12], we tried to develop models of black hole cosmology with modified Hawking's temperature relation as,

$$
\begin{aligned}
& T_{t} \cong \frac{\hbar c^{3}}{8 \pi k_{B} G \sqrt{M_{t} M_{p l}}} \\
& \text { where } M_{t} \cong\left(c^{3} / 2 G H_{t}\right)
\end{aligned}
$$

In those publications our aim was to couple Hubble parameter and cosmic temperature without cosmic rotation. In some situations, we were forced to assume the equality of Hubble parameter and angular velocity. By doing so, we could be able to match current Hubble radius and Hubble age. But, our idea of MOND's approach of dark matter analysis and advocated [43] Birch's cosmic angular velocity are not working for the case of $H_{0} \cong \omega_{0}$. Hence, in this paper, we are forced to accommodate $146.3\left(c / H_{0}\right)$ radius $146.3\left(1 / H_{0}\right)$. With future observations and advanced telescopes, it may be possible to see far distant galaxies. For example, the oldest stars in the Milky Way are nearly as old as the current universe itself. Based on this observation, by considering very old galaxies compared to Milky Way, there is a possibility of observing very old stars beyond our current observable radius which may help in understanding the actual cosmic radius and age.

\section{Discussion on Galactic Flat Rotation Curves and Galactic Dark Matter Estimation}

1) With reference to maximum possible (current) cosmic angular acceleration, MOND relation can be rewritten as follows.

$$
\begin{aligned}
& \left(V_{\text {Grot }}\right)_{0} \cong \sqrt[4]{G\left[27.28\left(M_{G v i s}\right)_{0}\right] c \omega_{0}} \\
& \text { where, }\left(\frac{1.2 \times 10^{-10} \mathrm{~m} \cdot \mathrm{sec}^{-2}}{c \omega_{0}}\right) \cong 27.28
\end{aligned}
$$

2) On comparison, percentage of dark mass in MOND model seems to be 
constant at $(26.28 / 27.28) \times 100=96.33 \%$ whereas in our approach, dark matter percentage increases with increasing (visible) mass and radius of galaxy. It is very interesting to note that, MOND's approach implicitly seems to support the cosmological estimation of $95 \%$ invisible matter and $5 \%$ visible matter. It needs further study.

3) By minimizing the errors in estimating the visible mass of galaxy and by properly choosing the effective radius of galaxy, accuracy can be improved in estimating the dark mass of a galaxy. Point to be noted is that, there is no correlation between photometric mass estimations and parametric mass estimations. Similarly, in some cases, including Milky Way, there is no correlation between MSTG mass estimations and MOND mass estimations. It needs a careful analysis.

4) Staring from the lowest massive galaxy, (DDO 154) to the highest massive galaxy (NGC 2841), dark mass seems to increase from 2.7 to 49.3 times respectively and needs further study. Applying this idea to Sun like stars, dark mass ratio is close to 0.0001 .

5) As per the recent studies [69], Virial mass of Milky Way is $1.28_{-0.48}^{+0.97} \times 10^{12} M_{\odot}$ and its corresponding upper limit is $2.25 \times 10^{12} M_{\odot}$. Based on proposed relations, for Milky Way [54], estimated flat rotation speed is $199.6 \mathrm{~km} / \mathrm{sec}$ and its corresponding total mass is $25.5 \times\left[10.6 \times 10^{10} M_{\odot}\right] \cong 2.7 \times 10^{12} M_{\odot}$. This is a good fit and strong support for our proposal. Based on relation (34), estimated angular velocity of whole Milky Way is $2.2 \times 10^{-17} \mathrm{rad} \cdot \mathrm{sec}^{-1}$. It is for observational testing and further study. Sun is located at $8 \mathrm{kpc}$ from the Milky Way center and is having a rotational period of 240 million years. So, for the whole Milky Way of radius $290 \mathrm{kpc}$, our proposal can be given a chance [70].

6) For Milky Way, its corresponding "visible" and "effective" radii are 11.5 $\mathrm{kpc}$ and $293.66 \mathrm{kpc}$. Corresponding geometric radius is $58.1 \mathrm{kpc}$. As per the observational data [71], for Milky Way, starting from a radius of $60 \mathrm{kpc}$, rotation speed seems to decrease gradually [72] [73]. Using the current dwarf galaxy population, very recently predicted [70] edge of the Milky Way halo is (292 \pm 61$)$ kpc. This is a very good support. See Table 1 for other galaxies.

7) In near future, by thoroughly studying the galactic dark mass distribution and corresponding deviations, variations in flat rotation speeds can be analyzed in a systematic approach. Very recent studies conducted on super spiral galaxies with Southern African Large Telescope (SALT) suggest high rotation speeds in the range of (240 to 570 ) $\mathrm{km} / \mathrm{sec}$ indicating a dynamical masse range of (0.6 to 4$) 10^{12} M_{\odot}$ [74]. It may be noted that, with a visible galactic mass of $(\sqrt{0.6 \times 4}) \times 10^{12} M_{\odot} \cong 1.55 \times 10^{12} M_{\odot}$, estimated flat rotation speed is $539 \mathrm{~km} / \mathrm{sec}$. This is a good support for our proposal pertaining to most massive galaxies having fast rotation speeds. Considering a visible mass of $(0.98 \pm 0.1)$ of $\log \left(M_{\text {dark }}\right)$ mentioned in Table 1 of Ref. [74], corresponding flat rotation speeds can be fitted well.

8) We are also working on developing alternative relations for estimating $\left(X_{\text {Gdark }}\right)_{0}$. On lower side, by studying the ultra faint dwarf galaxies it seems 
possible to fine tune $X_{\text {dark }}$.

9) Interesting point to be noted is that, for small galaxies whose mass is less than $3.5 \times 10^{38} \mathrm{~kg}$, their dark mass seems to be less than their visible mass. Whether it is, "correct or not", can be confirmed with their galactic rotational curves. For a galaxy of visible mass $10^{6} M_{\odot}$, galactic flat rotation speed seems to be $5.0 \mathrm{~km} / \mathrm{sec}$. It needs further investigation with respect to least massive galaxy, Segue 2. According to Evan N. Kirby et al. [75]: "Either Segue 2 would be the first of a vast class of new galaxies to be discovered with very low luminosities and very low dark matter content, or it would have to represent a rare case of a dark matter halo that is typically too small to host a galaxy but, for some reason, managed to form a small number of stars over at least 100 Myr." See Table 2 for low massive galaxies.

10) Relation (34) seems to be very simple in representation, easy to follow and simple to visualize and analyze MONDin approach connected with galactic structures and cosmic structure.

\section{Preparation of the Data Table 1}

By considering the available accurate flat rotation curves from SPARC data base [76] and considering relation (32) as a basic relation, galactic total matter can be estimated with the following relation.

$$
\left(M_{\text {Gtot }}\right)_{0} \cong \frac{\left(V_{\text {Grot }}\right)_{0}^{4}}{G c \omega_{0}}
$$

Based on relations (22), (25) and (26), we have,

$$
\left(M_{\text {Gtot }}\right)_{0} \cong\left(M_{\text {Gvis }}\right)_{0}+\left(M_{\text {Gdark }}\right)_{0} \cong\left(M_{\text {Gvis }}\right)_{0}+\frac{\sqrt[3]{\left(M_{\text {Gvis }}\right)_{0}}}{\sqrt{\left(M_{X}\right)_{0}}}
$$

We have developed a simple $\mathrm{C}++$ program to solve this relation and tried to estimate the galactic visible mass and dark mass approximately. In Table 1, we have presented the data.

Column-1: Galaxy name

Column-2: SPARC flat rotation speed excluding the error in $\mathrm{km} / \mathrm{sec}$

Column-3: Estimated total mass of galaxy in

Column-4: Estimated visible mass of galaxy in

Column-5: Estimated dark mass of galaxy in

Column-6: Estimated galactic dark mass percentage

Column-7: Estimated galactic visible radius in $\mathrm{kpc}$

Column-8: Geometric mean radius of galactic visible radius and effective radius in kpc

Column-9: Estimated galactic effective radius in kpc

Column-10: Fitted galactic visible mass density in $\mathrm{kg} / \mathrm{m}^{3}$ 
Table 1. Estimated galactic total mass, visible mass, dark mass, working radii and visible mass density.

\begin{tabular}{|c|c|c|c|c|c|c|c|c|c|}
\hline Galaxy & $\begin{array}{c}\text { Rotation } \\
\text { speed } \\
\mathrm{km} / \mathrm{sec}\end{array}$ & $\begin{array}{c}\text { Estimated } \\
\text { total mass } \\
10^{10} M_{\odot}\end{array}$ & $\begin{array}{c}\text { Estimated } \\
\text { visible mass } \\
10^{10} M_{\odot}\end{array}$ & $\begin{array}{c}\text { Estimated } \\
\text { dark mass } \\
10^{10} M_{\odot}\end{array}$ & $\begin{array}{c}\text { Dark } \\
\text { mass\% }\end{array}$ & $\begin{array}{c}\text { Estimated } \\
\text { visible radius } \\
\mathrm{kpc}\end{array}$ & $\begin{array}{l}\text { Estimated } \\
\text { geometric } \\
\text { radius } \mathrm{kpc}\end{array}$ & $\begin{array}{l}\text { Estimated } \\
\text { effective } \\
\text { radius kpc }\end{array}$ & $\begin{array}{c}\text { Fitted visible } \\
\text { mass density } \\
\mathrm{kg} / \mathrm{m}^{3}\end{array}$ \\
\hline D631-7 & 57.0 & 1.80 & 0.34 & 1.46 & 81.3 & 4.4 & 10.3 & 23.8 & $1.77 \mathrm{E}-27$ \\
\hline DDO064 & 46.1 & 0.77 & 0.18 & 0.59 & 76.3 & 3.7 & 7.6 & 15.6 & $2.47 \mathrm{E}-27$ \\
\hline DDO154 & 47.0 & 0.83 & 0.19 & 0.64 & 76.8 & 3.8 & 7.8 & 16.2 & $2.40 \mathrm{E}-27$ \\
\hline DDO161 & 66.3 & 3.29 & 0.51 & 2.77 & 84.4 & 5.0 & 12.7 & 32.2 & $1.39 \mathrm{E}-27$ \\
\hline DDO168 & 53.4 & 1.38 & 0.28 & 1.11 & 79.9 & 4.2 & 9.4 & 20.9 & $1.96 \mathrm{E}-27$ \\
\hline DDO170 & 60.0 & 2.21 & 0.39 & 1.82 & 82.4 & 4.6 & 11.1 & 26.4 & $1.63 \mathrm{E}-27$ \\
\hline ESO079-G014 & 175.0 & 159.63 & 7.43 & 152.20 & 95.3 & 10.4 & 48.4 & 224.2 & $2.88 \mathrm{E}-28$ \\
\hline ESO116-G012 & 109.1 & 24.11 & 2.05 & 22.07 & 91.5 & 7.4 & 25.4 & 87.2 & $6.25 \mathrm{E}-28$ \\
\hline ESO563-G021 & 314.6 & 1667.18 & 36.12 & 1631.07 & 97.8 & 15.7 & 106.7 & 724.7 & $1.09 \mathrm{E}-28$ \\
\hline F561-1 & 50.0 & 1.06 & 0.23 & 0.83 & 78.3 & 4.0 & 8.5 & 18.3 & $2.18 \mathrm{E}-27$ \\
\hline F563-V2 & 116.6 & 31.46 & 2.46 & 29.00 & 92.2 & 7.8 & 27.8 & 99.5 & $5.61 \mathrm{E}-28$ \\
\hline F568-V1 & 112.3 & 27.07 & 2.22 & 24.85 & 91.8 & 7.6 & 26.4 & 92.3 & $5.96 \mathrm{E}-28$ \\
\hline F571-8 & 139.7 & 64.82 & 4.03 & 60.80 & 93.8 & 8.9 & 35.6 & 142.9 & 4.17E-28 \\
\hline F571-V1 & 83.6 & 8.31 & 0.98 & 7.33 & 88.2 & 6.1 & 17.6 & 51.2 & $9.62 \mathrm{E}-28$ \\
\hline F574-1 & 97.8 & 15.57 & 1.52 & 14.06 & 90.3 & 6.8 & 21.9 & 70.0 & $7.46 \mathrm{E}-28$ \\
\hline F579-V1 & 112.1 & 26.88 & 2.21 & 24.67 & 91.8 & 7.6 & 26.4 & 92.0 & $5.98 \mathrm{E}-28$ \\
\hline F583-1 & 85.8 & 9.22 & 1.06 & 8.17 & 88.5 & 6.2 & 18.2 & 53.9 & $9.22 \mathrm{E}-28$ \\
\hline IC2574 & 66.4 & 3.31 & 0.52 & 2.79 & 84.4 & 5.0 & 12.8 & 32.3 & $1.39 \mathrm{E}-27$ \\
\hline IC4202 & 242.6 & 589.54 & 17.95 & 571.60 & 97.0 & 13.1 & 75.2 & 430.9 & $1.68 \mathrm{E}-28$ \\
\hline KK98-251 & 33.7 & 0.22 & 0.07 & 0.15 & 66.9 & 2.8 & 4.8 & 8.3 & $3.98 \mathrm{E}-27$ \\
\hline NGC0024 & 106.3 & 21.73 & 1.91 & 19.82 & 91.2 & 7.3 & 24.5 & 82.7 & $6.52 \mathrm{E}-28$ \\
\hline NGC0055 & 85.6 & 9.14 & 1.05 & 8.09 & 88.5 & 6.2 & 18.2 & 53.7 & $9.26 \mathrm{E}-28$ \\
\hline NGC0100 & 88.1 & 10.25 & 1.14 & 9.12 & 88.9 & 6.3 & 18.9 & 56.8 & $8.83 \mathrm{E}-28$ \\
\hline NGC0247 & 104.9 & 20.61 & 1.84 & 18.77 & 91.1 & 7.2 & 24.1 & 80.6 & $6.66 \mathrm{E}-28$ \\
\hline NGC0289 & 163.0 & 120.14 & 6.13 & 114.02 & 94.9 & 9.9 & 43.9 & 194.5 & $3.24 \mathrm{E}-28$ \\
\hline NGC0300 & 93.3 & 12.90 & 1.33 & 11.57 & 89.7 & 6.6 & 20.5 & 63.7 & $8.05 \mathrm{E}-28$ \\
\hline NGC0801 & 220.0 & 398.69 & 13.79 & 384.91 & 96.5 & 12.3 & 65.9 & 354.4 & $1.98 \mathrm{E}-28$ \\
\hline NGC0891 & 216.1 & 371.17 & 13.14 & 358.03 & 96.5 & 12.1 & 64.3 & 341.9 & $2.04 \mathrm{E}-28$ \\
\hline NGC1003 & 109.8 & 24.74 & 2.09 & 22.65 & 91.6 & 7.4 & 25.6 & 88.3 & $6.18 \mathrm{E}-28$ \\
\hline NGC1090 & 164.4 & 124.32 & 6.27 & 118.06 & 95.0 & 10.0 & 44.5 & 197.9 & $3.19 \mathrm{E}-28$ \\
\hline NGC1705 & 71.9 & 4.55 & 0.65 & 3.90 & 85.8 & 5.4 & 14.3 & 37.9 & $1.22 \mathrm{E}-27$ \\
\hline NGC2366 & 50.2 & 1.08 & 0.23 & 0.85 & 78.4 & 4.0 & 8.6 & 18.5 & $2.16 \mathrm{E}-27$ \\
\hline NGC2403 & 131.2 & 50.43 & 3.40 & 47.03 & 93.3 & 8.5 & 32.7 & 126.0 & $4.62 \mathrm{E}-28$ \\
\hline NGC2683 & 154.0 & 95.73 & 5.25 & 90.47 & 94.5 & 9.5 & 40.7 & 173.7 & $3.56 \mathrm{E}-28$ \\
\hline NGC2841 & 284.8 & 1119.72 & 27.64 & 1092.09 & 97.5 & 14.7 & 93.3 & 593.9 & $1.29 \mathrm{E}-28$ \\
\hline
\end{tabular}




\section{Continued}

\begin{tabular}{|c|c|c|c|c|c|c|c|c|c|}
\hline NGC2903 & 184.6 & 197.64 & 8.59 & 189.06 & 95.7 & 10.8 & 52.0 & 249.5 & $2.64 \mathrm{E}-28$ \\
\hline NGC2915 & 83.5 & 8.27 & 0.98 & 7.30 & 88.2 & 6.0 & 17.6 & 51.1 & $9.63 \mathrm{E}-28$ \\
\hline NGC2976 & 85.4 & 9.05 & 1.04 & 8.01 & 88.5 & 6.2 & 18.1 & 53.4 & $9.29 \mathrm{E}-28$ \\
\hline NGC2998 & 209.9 & 330.37 & 12.15 & 318.22 & 96.3 & 11.9 & 61.9 & 322.6 & $2.14 \mathrm{E}-28$ \\
\hline NGC3109 & 66.2 & 3.27 & 0.51 & 2.76 & 84.3 & 5.0 & 12.7 & 32.1 & $1.40 \mathrm{E}-27$ \\
\hline NGC3198 & 150.1 & 86.39 & 4.90 & 81.49 & 94.3 & 9.4 & 39.3 & 165.0 & $3.71 \mathrm{E}-28$ \\
\hline NGC3521 & 213.7 & 354.95 & 12.75 & 342.20 & 96.4 & 12.0 & 63.4 & 334.4 & $2.07 \mathrm{E}-28$ \\
\hline NGC3726 & 168.0 & 135.58 & 6.65 & 128.93 & 95.1 & 10.1 & 45.8 & 206.7 & $3.08 \mathrm{E}-28$ \\
\hline NGC3741 & 50.1 & 1.07 & 0.23 & 0.84 & 78.4 & 4.0 & 8.6 & 18.4 & $2.17 \mathrm{E}-27$ \\
\hline NGC3769 & 118.6 & 33.67 & 2.58 & 31.10 & 92.3 & 7.9 & 28.5 & 103.0 & $5.45 \mathrm{E}-28$ \\
\hline NGC3877 & 168.4 & 136.87 & 6.70 & 130.18 & 95.1 & 10.2 & 45.9 & 207.6 & $3.07 \mathrm{E}-28$ \\
\hline NGC3893 & 174.0 & 156.01 & 7.32 & 148.70 & 95.3 & 10.4 & 48.0 & 221.7 & $2.91 \mathrm{E}-28$ \\
\hline NGC3917 & 135.9 & 58.05 & 3.74 & 54.32 & 93.6 & 8.7 & 34.3 & 135.2 & $4.36 \mathrm{E}-28$ \\
\hline NGC3949 & 163.0 & 120.14 & 6.13 & 114.02 & 94.9 & 9.9 & 43.9 & 194.5 & $3.24 \mathrm{E}-28$ \\
\hline NGC3953 & 220.8 & 404.53 & 13.93 & 390.60 & 96.6 & 12.3 & 66.2 & 357.0 & $1.96 \mathrm{E}-28$ \\
\hline NGC3972 & 132.7 & 52.78 & 3.50 & 49.27 & 93.4 & 8.6 & 33.2 & 128.9 & $4.54 \mathrm{E}-28$ \\
\hline NGC3992 & 241.0 & 574.14 & 17.64 & 556.52 & 96.9 & 13.1 & 74.5 & 425.3 & $1.70 \mathrm{E}-28$ \\
\hline NGC4010 & 125.8 & 42.63 & 3.03 & 39.60 & 92.9 & 8.2 & 30.9 & 115.9 & $4.95 \mathrm{E}-28$ \\
\hline NGC4013 & 172.9 & 152.10 & 7.19 & 144.91 & 95.3 & 10.3 & 47.6 & 218.9 & $2.94 \mathrm{E}-28$ \\
\hline NGC4051 & 157.0 & 103.41 & 5.54 & 97.88 & 94.6 & 9.7 & 41.8 & 180.5 & $3.44 \mathrm{E}-28$ \\
\hline NGC4085 & 131.5 & 50.89 & 3.42 & 47.48 & 93.3 & 8.5 & 32.8 & 126.6 & $4.61 \mathrm{E}-28$ \\
\hline NGC4088 & 171.7 & 147.92 & 7.06 & 140.87 & 95.2 & 10.3 & 47.1 & 215.9 & $2.97 \mathrm{E}-28$ \\
\hline NGC4100 & 158.2 & 106.60 & 5.65 & 100.96 & 94.7 & 9.7 & 42.2 & 183.3 & $3.40 \mathrm{E}-28$ \\
\hline NGC4138 & 147.3 & 80.12 & 4.66 & 75.47 & 94.2 & 9.2 & 38.3 & 158.9 & $3.82 \mathrm{E}-28$ \\
\hline NGC4157 & 184.7 & 198.07 & 8.60 & 189.48 & 95.7 & 10.8 & 52.0 & 249.8 & $2.64 \mathrm{E}-28$ \\
\hline NGC4183 & 110.6 & 25.47 & 2.13 & 23.34 & 91.6 & 7.5 & 25.9 & 89.6 & $6.11 \mathrm{E}-28$ \\
\hline NGC4214 & 80.1 & 7.01 & 0.87 & 6.13 & 87.5 & 5.9 & 16.6 & 47.0 & $1.03 \mathrm{E}-27$ \\
\hline NGC4217 & 181.3 & 183.88 & 8.18 & 175.71 & 95.6 & 10.7 & 50.8 & 240.7 & $2.72 \mathrm{E}-28$ \\
\hline NGC4559 & 121.2 & 36.72 & 2.74 & 33.99 & 92.6 & 8.0 & 29.4 & 107.6 & $5.26 \mathrm{E}-28$ \\
\hline NGC5005 & 262.2 & 804.41 & 22.13 & 782.29 & 97.2 & 13.8 & 83.5 & 503.4 & $1.48 \mathrm{E}-28$ \\
\hline NGC5033 & 194.2 & 242.07 & 9.85 & 232.23 & 95.9 & 11.2 & 55.7 & 276.1 & $2.43 \mathrm{E}-28$ \\
\hline NGC5055 & 179.0 & 174.73 & 7.90 & 166.83 & 95.5 & 10.6 & 49.9 & 234.6 & $2.78 \mathrm{E}-28$ \\
\hline NGC5371 & 209.5 & 327.86 & 12.09 & 315.78 & 96.3 & 11.8 & 61.7 & 321.4 & $2.14 \mathrm{E}-28$ \\
\hline NGC5585 & 90.3 & 11.32 & 1.22 & 10.10 & 89.2 & 6.4 & 19.6 & 59.7 & $8.49 \mathrm{E}-28$ \\
\hline NGC5907 & 215.0 & 363.67 & 12.96 & 350.71 & 96.4 & 12.1 & 63.9 & 338.5 & $2.05 \mathrm{E}-28$ \\
\hline NGC5985 & 293.6 & 1264.66 & 30.00 & 1234.67 & 97.6 & 15.0 & 97.2 & 631.2 & $1.23 \mathrm{E}-28$ \\
\hline NGC6015 & 154.1 & 95.98 & 5.26 & 90.71 & 94.5 & 9.5 & 40.7 & 173.9 & $3.55 \mathrm{E}-28$ \\
\hline NGC6195 & 251.7 & 683.10 & 19.83 & 663.28 & 97.1 & 13.5 & 79.0 & 463.9 & $1.58 \mathrm{E}-28$ \\
\hline NGC6503 & 116.3 & 31.14 & 2.44 & 28.69 & 92.2 & 7.8 & 27.7 & 99.0 & $5.63 \mathrm{E}-28$ \\
\hline
\end{tabular}


Continued

\begin{tabular}{|c|c|c|c|c|c|c|c|c|c|}
\hline NGC6674 & 241.3 & 577.00 & 17.70 & 559.32 & 96.9 & 13.1 & 74.7 & 426.3 & $1.70 \mathrm{E}-28$ \\
\hline NGC6946 & 158.9 & 108.50 & 5.72 & 102.78 & 94.7 & 9.7 & 42.4 & 184.9 & $3.38 \mathrm{E}-28$ \\
\hline NGC7331 & 239.0 & 555.32 & 17.24 & 538.08 & 96.9 & 13.0 & 73.7 & 418.2 & $1.72 \mathrm{E}-28$ \\
\hline NGC7814 & 218.9 & 390.78 & 13.61 & 377.18 & 96.5 & 12.2 & 65.5 & 350.9 & $1.99 \mathrm{E}-28$ \\
\hline PGC51017 & 18.6 & 0.02 & 0.01 & 0.01 & 44.5 & 1.4 & 1.9 & 2.5 & $9.31 \mathrm{E}-27$ \\
\hline UGC00128 & 129.3 & 47.57 & 3.26 & 44.31 & 93.1 & 8.4 & 32.1 & 122.4 & $4.73 \mathrm{E}-28$ \\
\hline UGC00731 & 73.3 & 4.91 & 0.68 & 4.23 & 86.1 & 5.5 & 14.7 & 39.3 & $1.19 \mathrm{E}-27$ \\
\hline UGC01230 & 103.7 & 19.68 & 1.78 & 17.90 & 90.9 & 7.1 & 23.7 & 78.7 & $6.78 \mathrm{E}-28$ \\
\hline UGC01281 & 55.2 & 1.58 & 0.31 & 1.27 & 80.6 & 4.3 & 9.8 & 22.3 & $1.86 \mathrm{E}-27$ \\
\hline UGC02259 & 86.2 & 9.40 & 1.07 & 8.33 & 88.6 & 6.2 & 18.4 & 54.4 & $9.15 \mathrm{E}-28$ \\
\hline UGC02487 & 332.0 & 2067.77 & 41.74 & 2026.04 & 98.0 & 16.3 & 114.7 & 807.1 & $1.00 \mathrm{E}-28$ \\
\hline UGC02885 & 289.5 & 1195.48 & 28.89 & 1166.61 & 97.6 & 14.8 & 95.4 & 613.7 & $1.26 \mathrm{E}-28$ \\
\hline UGC02916 & 182.7 & 189.63 & 8.35 & 181.28 & 95.6 & 10.8 & 51.3 & 244.4 & $2.68 \mathrm{E}-28$ \\
\hline UGC02953 & 264.9 & 838.06 & 22.75 & 815.33 & 97.3 & 13.9 & 84.7 & 513.8 & $1.45 \mathrm{E}-28$ \\
\hline UGC03205 & 219.6 & 395.80 & 13.73 & 382.08 & 96.5 & 12.2 & 65.8 & 353.1 & $1.98 \mathrm{E}-28$ \\
\hline UGC03546 & 196.9 & 255.82 & 10.22 & 245.60 & 96.0 & 11.3 & 56.7 & 283.9 & $2.37 \mathrm{E}-28$ \\
\hline UGC03580 & 126.2 & 43.17 & 3.05 & 40.12 & 92.9 & 8.3 & 31.0 & 116.6 & $4.93 \mathrm{E}-28$ \\
\hline UGC04278 & 91.4 & 11.88 & 1.26 & 10.62 & 89.4 & 6.5 & 19.9 & 61.2 & $8.33 \mathrm{E}-28$ \\
\hline UGC04305 & 34.5 & 0.24 & 0.08 & 0.16 & 67.7 & 2.8 & 5.0 & 8.7 & $3.83 \mathrm{E}-27$ \\
\hline UGC04325 & 90.9 & 11.62 & 1.24 & 10.38 & 89.3 & 6.5 & 19.8 & 60.5 & $8.40 \mathrm{E}-28$ \\
\hline UGC04499 & 72.8 & 4.78 & 0.67 & 4.11 & 86.0 & 5.4 & 14.5 & 38.8 & $1.20 \mathrm{E}-27$ \\
\hline UGC05005 & 98.9 & 16.28 & 1.57 & 14.72 & 90.4 & 6.9 & 22.2 & 71.6 & $7.33 \mathrm{E}-28$ \\
\hline UGC05253 & 213.7 & 355.02 & 12.75 & 342.27 & 96.4 & 12.0 & 63.4 & 334.4 & $2.07 \mathrm{E}-28$ \\
\hline UGC05716 & 73.1 & 4.86 & 0.68 & 4.18 & 86.1 & 5.4 & 14.6 & 39.1 & $1.19 \mathrm{E}-27$ \\
\hline UGC05721 & 79.7 & 6.87 & 0.86 & 6.01 & 87.5 & 5.8 & 16.5 & 46.5 & $1.04 \mathrm{E}-27$ \\
\hline UGC05986 & 113.0 & 27.75 & 2.26 & 25.50 & 91.9 & 7.6 & 26.7 & 93.5 & $5.90 \mathrm{E}-28$ \\
\hline UGC06399 & 85.0 & 8.88 & 1.03 & 7.86 & 88.4 & 6.1 & 18.0 & 52.9 & $9.36 \mathrm{E}-28$ \\
\hline UGC06446 & 82.2 & 7.77 & 0.94 & 6.83 & 87.9 & 6.0 & 17.2 & 49.5 & $9.88 \mathrm{E}-28$ \\
\hline UGC06614 & 199.8 & 271.23 & 10.64 & 260.60 & 96.1 & 11.5 & 57.9 & 292.3 & $2.32 \mathrm{E}-28$ \\
\hline UGC06628 & 41.8 & 0.52 & 0.14 & 0.38 & 73.6 & 3.4 & 6.6 & 12.8 & $2.87 \mathrm{E}-27$ \\
\hline UGC06667 & 83.8 & 8.39 & 0.99 & 7.41 & 88.2 & 6.1 & 17.7 & 51.4 & $9.58 \mathrm{E}-28$ \\
\hline UGC06786 & 219.4 & 394.36 & 13.69 & 380.68 & 96.5 & 12.2 & 65.7 & 352.5 & $1.99 \mathrm{E}-28$ \\
\hline UGC06787 & 248.1 & 644.85 & 19.07 & 625.79 & 97.0 & 13.3 & 77.5 & 450.7 & $1.62 \mathrm{E}-28$ \\
\hline UGC06818 & 71.2 & 4.37 & 0.63 & 3.75 & 85.6 & 5.3 & 14.1 & 37.1 & $1.24 \mathrm{E}-27$ \\
\hline UGC06917 & 108.7 & 23.76 & 2.03 & 21.73 & 91.5 & 7.4 & 25.3 & 86.5 & $6.28 \mathrm{E}-28$ \\
\hline UGC06923 & 79.6 & 6.83 & 0.86 & 5.98 & 87.4 & 5.8 & 16.4 & 46.4 & $1.04 \mathrm{E}-27$ \\
\hline UGC06930 & 107.2 & 22.48 & 1.95 & 20.52 & 91.3 & 7.3 & 24.8 & 84.1 & $6.43 \mathrm{E}-28$ \\
\hline UGC06973 & 174.2 & 156.73 & 7.34 & 149.39 & 95.3 & 10.4 & 48.1 & 222.2 & $2.90 \mathrm{E}-28$ \\
\hline UGC06983 & 109.0 & 24.02 & 2.05 & 21.98 & 91.5 & 7.4 & 25.4 & 87.0 & $6.26 \mathrm{E}-28$ \\
\hline
\end{tabular}




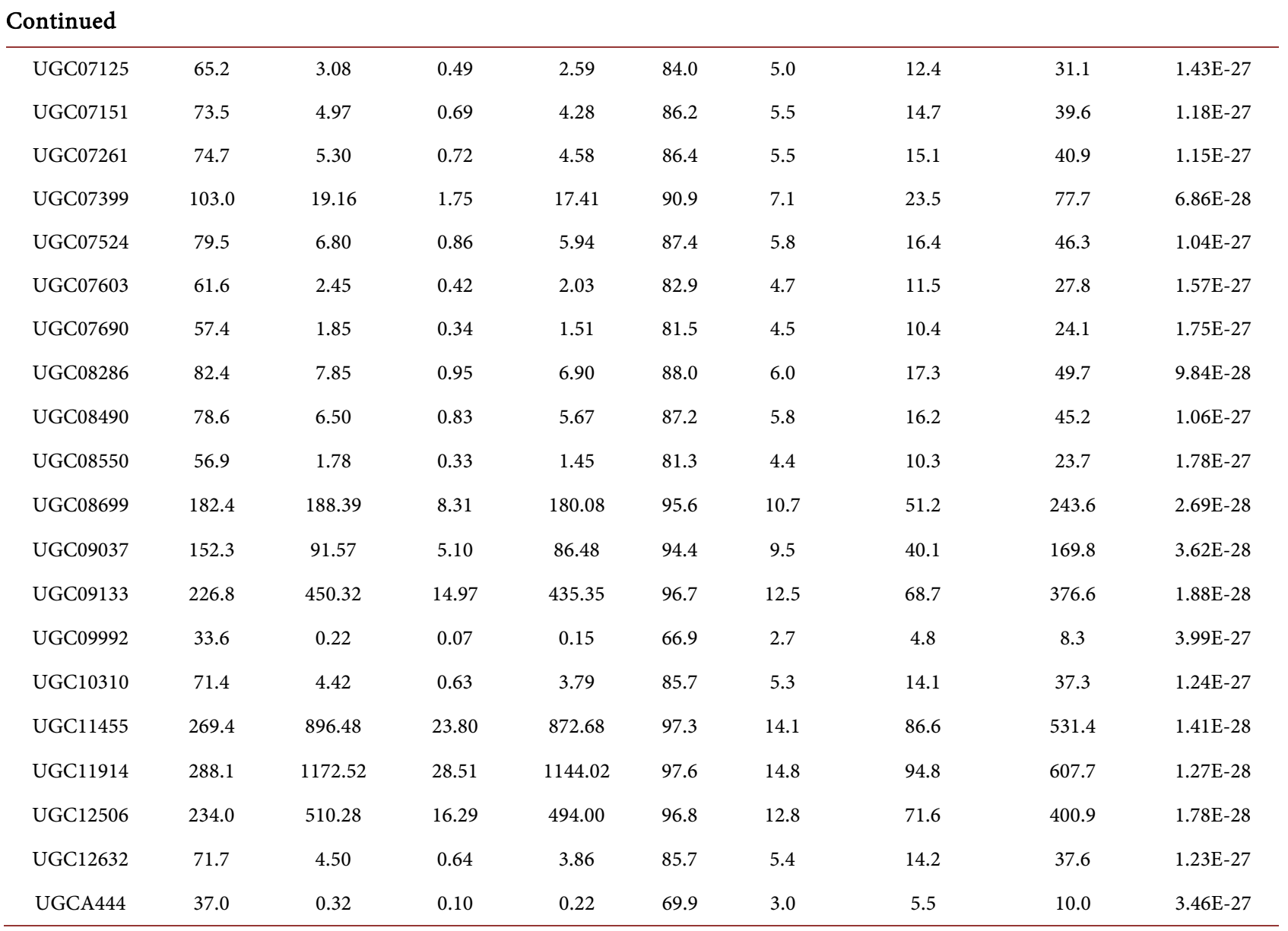

\section{Preparation of the Data Table 2}

For low massive galaxies whose mass is less than $\left(M_{X}\right) \cong 3.523 \times 10^{38} \mathrm{~kg}$, we assume their visible mass as,

$$
\begin{aligned}
& \left(M_{\text {Gvis }}\right)_{0} \cong n \times \alpha \times\left(M_{X}\right)_{0} \\
& \text { where, } n=1,2,3, \cdots, 137 \\
& \alpha=\text { Fine structure constant } \cong 7.297353 \times 10^{-3}
\end{aligned}
$$

Note: No change in estimation of other parameters.

Column-1: Assumed Alpha multiple

Column-2: Assumed visible mass of galaxy in $10^{6} M_{\odot}$

Column-3: Estimated dark mass of galaxy in $10^{6} \mathrm{M}_{\odot}$

Column-4: Estimated total mass of galaxy in $10^{6} \mathrm{M}_{\odot}$

Column-5: Estimated flat rotation speed of galaxy in $\mathrm{km} / \mathrm{sec}$

Column-6: Estimated galactic dark mass percentage

Column-7: Estimated galactic visible radius in $\mathrm{kpc}$

Column-8: Geometric mean radius of galactic visible radius and effective radius

Column-9: Estimated galactic effective radius in $\mathrm{kpc}$

Column-10: Fitted galactic visible mass density in $\mathrm{kg} / \mathrm{m}^{3}$ 
Table 2. Assumed galactic visible mass and estimated dark mass, total mass, working radii and visible mass density of low massive galaxies.

\begin{tabular}{|c|c|c|c|c|c|c|c|c|c|}
\hline $\begin{array}{l}\text { Alpha } \\
\text { multiple (n) }\end{array}$ & $\begin{array}{c}\text { Assumed } \\
\text { Visible mass } \\
10^{6} M_{\odot}\end{array}$ & $\begin{array}{c}\text { Estimated } \\
\text { dark mass } \\
10^{6} M_{\odot}\end{array}$ & $\begin{array}{c}\text { Estimated } \\
\text { total mass } \\
10^{6} M_{\odot}\end{array}$ & $\begin{array}{c}\text { Estimated flat } \\
\text { rotation speed } \\
\mathrm{km} / \mathrm{sec}\end{array}$ & $\begin{array}{c}\text { Dark } \\
\text { matter \% }\end{array}$ & $\begin{array}{c}\text { Estimated } \\
\text { visible radius } \\
\text { kpc }\end{array}$ & $\begin{array}{l}\text { Estimated } \\
\text { geometric } \\
\text { radius kpc }\end{array}$ & $\begin{array}{l}\text { Estimated } \\
\text { effective } \\
\text { radius kpc }\end{array}$ & $\begin{array}{c}\text { Fitted visible } \\
\text { mass density } \\
\mathrm{kg} / \mathrm{m}^{3}\end{array}$ \\
\hline (1) & (2) & (3) & (4) & (5) & (6) & (7) & (8) & (9) & (10) \\
\hline 1 & 1.29 & 0.11 & 1.40 & 5.36 & 7.87 & 0.19 & 0.20 & 0.21 & $4.19 \mathrm{E}-26$ \\
\hline 2 & 2.59 & 0.31 & 2.90 & 6.42 & 10.78 & 0.27 & 0.29 & 0.30 & $3.44 \mathrm{E}-26$ \\
\hline 3 & 3.88 & 0.57 & 4.45 & 7.15 & 12.89 & 0.33 & 0.35 & 0.37 & $3.05 \mathrm{E}-26$ \\
\hline 4 & 5.17 & 0.88 & 6.05 & 7.72 & 14.59 & 0.37 & 0.40 & 0.44 & $2.8 \mathrm{E}-26$ \\
\hline 5 & 6.46 & 1.23 & 7.70 & 8.20 & 16.04 & 0.41 & 0.45 & 0.49 & $2.61 \mathrm{E}-26$ \\
\hline 6 & 7.76 & 1.62 & 9.38 & 8.62 & 17.30 & 0.45 & 0.49 & 0.54 & $2.47 \mathrm{E}-26$ \\
\hline 7 & 9.05 & 2.04 & 11.09 & 8.99 & 18.43 & 0.48 & 0.53 & 0.59 & $2.35 \mathrm{E}-26$ \\
\hline 8 & 10.34 & 2.50 & 12.84 & 9.32 & 19.46 & 0.51 & 0.57 & 0.64 & $2.25 \mathrm{E}-26$ \\
\hline 9 & 11.63 & 2.98 & 14.61 & 9.63 & 20.40 & 0.54 & 0.61 & 0.68 & $2.17 \mathrm{E}-26$ \\
\hline 10 & 12.93 & 3.49 & 16.42 & 9.91 & 21.27 & 0.57 & 0.64 & 0.72 & $2.09 \mathrm{E}-26$ \\
\hline 11 & 14.22 & 4.03 & 18.25 & 10.18 & 22.08 & 0.59 & 0.67 & 0.76 & $2.03 \mathrm{E}-26$ \\
\hline 12 & 15.51 & 4.59 & 20.10 & 10.42 & 22.83 & 0.61 & 0.70 & 0.80 & $1.97 \mathrm{E}-26$ \\
\hline 13 & 16.80 & 5.18 & 21.98 & 10.66 & 23.55 & 0.64 & 0.73 & 0.83 & $1.92 \mathrm{E}-26$ \\
\hline 14 & 18.10 & 5.78 & 23.88 & 10.88 & 24.22 & 0.66 & 0.76 & 0.87 & $1.87 \mathrm{E}-26$ \\
\hline 15 & 19.39 & 6.41 & 25.80 & 11.10 & 24.86 & 0.68 & 0.78 & 0.90 & $1.83 \mathrm{E}-26$ \\
\hline 16 & 20.68 & 7.07 & 27.75 & 11.30 & 25.47 & 0.70 & 0.81 & 0.93 & $1.79 \mathrm{E}-26$ \\
\hline 17 & 21.97 & 7.74 & 29.71 & 11.49 & 26.05 & 0.72 & 0.83 & 0.97 & $1.75 \mathrm{E}-26$ \\
\hline 18 & 23.27 & 8.43 & 31.70 & 11.68 & 26.60 & 0.73 & 0.86 & 1.00 & $1.71 \mathrm{E}-26$ \\
\hline 19 & 24.56 & 9.14 & 33.70 & 11.86 & 27.13 & 0.75 & 0.88 & 1.03 & $1.68 \mathrm{E}-26$ \\
\hline 20 & 25.85 & 9.88 & 35.73 & 12.04 & 27.64 & 0.77 & 0.90 & 1.06 & $1.65 \mathrm{E}-26$ \\
\hline 21 & 27.14 & 10.63 & 37.77 & 12.21 & 28.13 & 0.78 & 0.92 & 1.09 & $1.62 \mathrm{E}-26$ \\
\hline 22 & 28.44 & 11.39 & 39.83 & 12.37 & 28.61 & 0.80 & 0.95 & 1.12 & $1.6 \mathrm{E}-26$ \\
\hline 23 & 29.73 & 12.18 & 41.91 & 12.53 & 29.06 & 0.82 & 0.97 & 1.15 & $1.57 \mathrm{E}-26$ \\
\hline 24 & 31.02 & 12.98 & 44.00 & 12.68 & 29.50 & 0.83 & 0.99 & 1.18 & $1.55 \mathrm{E}-26$ \\
\hline 25 & 32.31 & 13.80 & 46.12 & 12.83 & 29.93 & 0.84 & 1.01 & 1.21 & $1.53 \mathrm{E}-26$ \\
\hline 26 & 33.61 & 14.64 & 48.24 & 12.98 & 30.34 & 0.86 & 1.03 & 1.23 & $1.5 \mathrm{E}-26$ \\
\hline 27 & 34.90 & 15.49 & 50.39 & 13.12 & 30.74 & 0.87 & 1.05 & 1.26 & $1.48 \mathrm{E}-26$ \\
\hline 28 & 36.19 & 16.36 & 52.55 & 13.26 & 31.13 & 0.89 & 1.07 & 1.29 & $1.46 \mathrm{E}-26$ \\
\hline 29 & 37.48 & 17.24 & 54.73 & 13.39 & 31.51 & 0.90 & 1.09 & 1.31 & $1.44 \mathrm{E}-26$ \\
\hline 30 & 38.78 & 18.14 & 56.92 & 13.52 & 31.88 & 0.91 & 1.11 & 1.34 & $1.43 \mathrm{E}-26$ \\
\hline 31 & 40.07 & 19.06 & 59.13 & 13.65 & 32.23 & 0.92 & 1.12 & 1.36 & $1.41 \mathrm{E}-26$ \\
\hline 32 & 41.36 & 19.99 & 61.35 & 13.78 & 32.58 & 0.94 & 1.14 & 1.39 & $1.39 \mathrm{E}-26$ \\
\hline 33 & 42.65 & 20.93 & 63.59 & 13.90 & 32.92 & 0.95 & 1.16 & 1.42 & $1.38 \mathrm{E}-26$ \\
\hline
\end{tabular}




\section{Continued}

\begin{tabular}{|c|c|c|c|c|c|c|c|c|c|}
\hline 34 & 43.95 & 21.89 & 65.84 & 14.02 & 33.25 & 0.96 & 1.18 & 1.44 & $1.36 \mathrm{E}-26$ \\
\hline 35 & 45.24 & 22.86 & 68.10 & 14.14 & 33.57 & 0.97 & 1.19 & 1.46 & $1.35 \mathrm{E}-26$ \\
\hline 36 & 46.53 & 23.85 & 70.38 & 14.26 & 33.89 & 0.98 & 1.21 & 1.49 & $1.33 \mathrm{E}-26$ \\
\hline 37 & 47.82 & 24.85 & 72.67 & 14.38 & 34.19 & 1.00 & 1.23 & 1.51 & $1.32 \mathrm{E}-26$ \\
\hline 38 & 49.12 & 25.86 & 74.98 & 14.49 & 34.49 & 1.01 & 1.24 & 1.54 & $1.31 \mathrm{E}-26$ \\
\hline 39 & 50.41 & 26.89 & 77.30 & 14.60 & 34.79 & 1.02 & 1.26 & 1.56 & $1.29 \mathrm{E}-26$ \\
\hline 40 & 51.70 & 27.93 & 79.63 & 14.71 & 35.08 & 1.03 & 1.28 & 1.58 & $1.28 \mathrm{E}-26$ \\
\hline 41 & 52.99 & 28.99 & 81.98 & 14.81 & 35.36 & 1.04 & 1.29 & 1.61 & $1.27 \mathrm{E}-26$ \\
\hline 42 & 54.29 & 30.05 & 84.34 & 14.92 & 35.63 & 1.05 & 1.31 & 1.63 & $1.26 \mathrm{E}-26$ \\
\hline 43 & 55.58 & 31.13 & 86.71 & 15.02 & 35.90 & 1.06 & 1.32 & 1.65 & $1.25 \mathrm{E}-26$ \\
\hline 44 & 56.87 & 32.23 & 89.10 & 15.13 & 36.17 & 1.07 & 1.34 & 1.68 & $1.23 \mathrm{E}-26$ \\
\hline 45 & 58.16 & 33.33 & 91.49 & 15.23 & 36.43 & 1.08 & 1.35 & 1.70 & $1.22 \mathrm{E}-26$ \\
\hline 46 & 59.46 & 34.45 & 93.90 & 15.33 & 36.68 & 1.09 & 1.37 & 1.72 & $1.21 \mathrm{E}-26$ \\
\hline 47 & 60.75 & 35.58 & 96.33 & 15.42 & 36.93 & 1.10 & 1.38 & 1.74 & $1.2 \mathrm{E}-26$ \\
\hline 48 & 62.04 & 36.72 & 98.76 & 15.52 & 37.18 & 1.11 & 1.40 & 1.76 & $1.19 \mathrm{E}-26$ \\
\hline 49 & 63.33 & 37.87 & 101.21 & 15.62 & 37.42 & 1.12 & 1.41 & 1.79 & $1.18 \mathrm{E}-26$ \\
\hline 50 & 64.63 & 39.04 & 103.66 & 15.71 & 37.66 & 1.13 & 1.43 & 1.81 & $1.17 \mathrm{E}-26$ \\
\hline 51 & 65.92 & 40.21 & 106.13 & 15.80 & 37.89 & 1.14 & 1.44 & 1.83 & $1.17 \mathrm{E}-26$ \\
\hline 52 & 67.21 & 41.40 & 108.62 & 15.89 & 38.12 & 1.14 & 1.46 & 1.85 & $1.16 \mathrm{E}-26$ \\
\hline 53 & 68.50 & 42.60 & 111.11 & 15.98 & 38.34 & 1.15 & 1.47 & 1.87 & $1.15 \mathrm{E}-26$ \\
\hline 54 & 69.80 & 43.81 & 113.61 & 16.07 & 38.57 & 1.16 & 1.48 & 1.89 & $1.14 \mathrm{E}-26$ \\
\hline 55 & 71.09 & 45.04 & 116.13 & 16.16 & 38.78 & 1.17 & 1.50 & 1.91 & $1.13 \mathrm{E}-26$ \\
\hline 56 & 72.38 & 46.27 & 118.65 & 16.25 & 39.00 & 1.18 & 1.51 & 1.93 & $1.12 \mathrm{E}-26$ \\
\hline 57 & 73.67 & 47.52 & 121.19 & 16.34 & 39.21 & 1.19 & 1.52 & 1.95 & $1.12 \mathrm{E}-26$ \\
\hline 58 & 74.97 & 48.77 & 123.74 & 16.42 & 39.42 & 1.20 & 1.54 & 1.97 & $1.11 \mathrm{E}-26$ \\
\hline 59 & 76.26 & 50.04 & 126.30 & 16.50 & 39.62 & 1.20 & 1.55 & 1.99 & $1.1 \mathrm{E}-26$ \\
\hline 60 & 77.55 & 51.32 & 128.87 & 16.59 & 39.82 & 1.21 & 1.56 & 2.01 & $1.09 \mathrm{E}-26$ \\
\hline 61 & 78.84 & 52.60 & 131.45 & 16.67 & 40.02 & 1.22 & 1.58 & 2.03 & $1.09 \mathrm{E}-26$ \\
\hline 62 & 80.14 & 53.90 & 134.04 & 16.75 & 40.21 & 1.23 & 1.59 & 2.05 & $1.08 \mathrm{E}-26$ \\
\hline 63 & 81.43 & 55.21 & 136.64 & 16.83 & 40.41 & 1.24 & 1.60 & 2.07 & $1.07 \mathrm{E}-26$ \\
\hline 64 & 82.72 & 56.53 & 139.26 & 16.91 & 40.60 & 1.24 & 1.61 & 2.09 & $1.07 \mathrm{E}-26$ \\
\hline 65 & 84.01 & 57.86 & 141.88 & 16.99 & 40.78 & 1.25 & 1.63 & 2.11 & $1.06 \mathrm{E}-26$ \\
\hline 66 & 85.31 & 59.20 & 144.51 & 17.07 & 40.97 & 1.26 & 1.64 & 2.13 & $1.05 \mathrm{E}-26$ \\
\hline 67 & 86.60 & 60.55 & 147.15 & 17.15 & 41.15 & 1.27 & 1.65 & 2.15 & $1.05 \mathrm{E}-26$ \\
\hline 68 & 87.89 & 61.91 & 149.81 & 17.22 & 41.33 & 1.27 & 1.66 & 2.17 & $1.04 \mathrm{E}-26$ \\
\hline 69 & 89.19 & 63.28 & 152.47 & 17.30 & 41.51 & 1.28 & 1.68 & 2.19 & $1.03 \mathrm{E}-26$ \\
\hline 70 & 90.48 & 64.67 & 155.14 & 17.38 & 41.68 & 1.29 & 1.69 & 2.21 & $1.03 \mathrm{E}-26$ \\
\hline
\end{tabular}




\section{Continued}

\begin{tabular}{|c|c|c|c|c|c|c|c|c|c|}
\hline 71 & 91.77 & 66.06 & 157.83 & 17.45 & 41.85 & 1.30 & 1.70 & 2.23 & $1.02 \mathrm{E}-26$ \\
\hline 72 & 93.06 & 67.46 & 160.52 & 17.52 & 42.02 & 1.30 & 1.71 & 2.25 & $1.02 \mathrm{E}-26$ \\
\hline 73 & 94.36 & 68.87 & 163.22 & 17.60 & 42.19 & 1.31 & 1.72 & 2.27 & $1.01 \mathrm{E}-26$ \\
\hline 74 & 95.65 & 70.29 & 165.94 & 17.67 & 42.36 & 1.32 & 1.74 & 2.29 & $1.00 \mathrm{E}-26$ \\
\hline 75 & 96.94 & 71.72 & 168.66 & 17.74 & 42.52 & 1.32 & 1.75 & 2.30 & $9.99 \mathrm{E}-27$ \\
\hline 76 & 98.23 & 73.16 & 171.39 & 17.81 & 42.68 & 1.33 & 1.76 & 2.32 & $9.93 \mathrm{E}-27$ \\
\hline 77 & 99.53 & 74.60 & 174.13 & 17.88 & 42.84 & 1.34 & 1.77 & 2.34 & $9.88 \mathrm{E}-27$ \\
\hline 78 & 100.82 & 76.06 & 176.88 & 17.95 & 43.00 & 1.35 & 1.78 & 2.36 & $9.83 \mathrm{E}-27$ \\
\hline 79 & 102.11 & 77.53 & 179.64 & 18.02 & 43.16 & 1.35 & 1.79 & 2.38 & $9.78 \mathrm{E}-27$ \\
\hline 80 & 103.40 & 79.01 & 182.41 & 18.09 & 43.31 & 1.36 & 1.80 & 2.40 & $9.73 \mathrm{E}-27$ \\
\hline 81 & 104.70 & 80.49 & 185.19 & 18.16 & 43.47 & 1.37 & 1.82 & 2.42 & $9.68 \mathrm{E}-27$ \\
\hline 82 & 105.99 & 81.99 & 187.98 & 18.23 & 43.62 & 1.37 & 1.83 & 2.43 & $9.63 \mathrm{E}-27$ \\
\hline 83 & 107.28 & 83.49 & 190.77 & 18.30 & 43.77 & 1.38 & 1.84 & 2.45 & $9.58 \mathrm{E}-27$ \\
\hline 84 & 108.57 & 85.01 & 193.58 & 18.36 & 43.91 & 1.39 & 1.85 & 2.47 & $9.53 \mathrm{E}-27$ \\
\hline 85 & 109.87 & 86.53 & 196.39 & 18.43 & 44.06 & 1.39 & 1.86 & 2.49 & $9.49 \mathrm{E}-27$ \\
\hline 86 & 111.16 & 88.06 & 199.22 & 18.50 & 44.20 & 1.40 & 1.87 & 2.51 & $9.44 \mathrm{E}-27$ \\
\hline 87 & 112.45 & 89.60 & 202.05 & 18.56 & 44.35 & 1.40 & 1.88 & 2.52 & $9.39 \mathrm{E}-27$ \\
\hline 88 & 113.74 & 91.15 & 204.89 & 18.63 & 44.49 & 1.41 & 1.89 & 2.54 & $9.35 \mathrm{E}-27$ \\
\hline 89 & 115.04 & 92.71 & 207.74 & 18.69 & 44.63 & 1.42 & 1.90 & 2.56 & $9.31 \mathrm{E}-27$ \\
\hline 90 & 116.33 & 94.27 & 210.60 & 18.76 & 44.76 & 1.42 & 1.91 & 2.58 & $9.26 \mathrm{E}-27$ \\
\hline 91 & 117.62 & 95.85 & 213.47 & 18.82 & 44.90 & 1.43 & 1.92 & 2.59 & $9.22 \mathrm{E}-27$ \\
\hline 92 & 118.91 & 97.43 & 216.35 & 18.88 & 45.04 & 1.43 & 1.94 & 2.61 & $9.18 \mathrm{E}-27$ \\
\hline 93 & 120.21 & 99.03 & 219.23 & 18.94 & 45.17 & 1.44 & 1.95 & 2.63 & $9.14 \mathrm{E}-27$ \\
\hline 94 & 121.50 & 100.63 & 222.13 & 19.01 & 45.30 & 1.45 & 1.96 & 2.65 & $9.1 \mathrm{E}-27$ \\
\hline 95 & 122.79 & 102.24 & 225.03 & 19.07 & 45.43 & 1.45 & 1.97 & 2.66 & $9.06 \mathrm{E}-27$ \\
\hline 96 & 124.08 & 103.86 & 227.94 & 19.13 & 45.56 & 1.46 & 1.98 & 2.68 & $9.02 \mathrm{E}-27$ \\
\hline 97 & 125.38 & 105.48 & 230.86 & 19.19 & 45.69 & 1.46 & 1.99 & 2.70 & $8.98 \mathrm{E}-27$ \\
\hline 98 & 126.67 & 107.12 & 233.79 & 19.25 & 45.82 & 1.47 & 2.00 & 2.71 & $8.94 \mathrm{E}-27$ \\
\hline 99 & 127.96 & 108.76 & 236.72 & 19.31 & 45.94 & 1.48 & 2.01 & 2.73 & $8.9 \mathrm{E}-27$ \\
\hline 100 & 129.25 & 110.41 & 239.67 & 19.37 & 46.07 & 1.48 & 2.02 & 2.75 & $8.86 \mathrm{E}-27$ \\
\hline 101 & 130.55 & 112.08 & 242.62 & 19.43 & 46.19 & 1.49 & 2.03 & 2.76 & $8.82 \mathrm{E}-27$ \\
\hline 102 & 131.84 & 113.74 & 245.58 & 19.49 & 46.32 & 1.49 & 2.04 & 2.78 & $8.79 \mathrm{E}-27$ \\
\hline 103 & 133.13 & 115.42 & 248.55 & 19.55 & 46.44 & 1.50 & 2.05 & 2.80 & $8.75 \mathrm{E}-27$ \\
\hline 104 & 134.42 & 117.11 & 251.53 & 19.61 & 46.56 & 1.50 & 2.06 & 2.81 & $8.72 \mathrm{E}-27$ \\
\hline 105 & 135.72 & 118.80 & 254.52 & 19.66 & 46.68 & 1.51 & 2.07 & 2.83 & $8.68 \mathrm{E}-27$ \\
\hline 106 & 137.01 & 120.50 & 257.51 & 19.72 & 46.79 & 1.52 & 2.08 & 2.85 & $8.65 \mathrm{E}-27$ \\
\hline 107 & 138.30 & 122.21 & 260.51 & 19.78 & 46.91 & 1.52 & 2.09 & 2.86 & $8.61 \mathrm{E}-27$ \\
\hline
\end{tabular}




\section{Continued}

\begin{tabular}{|c|c|c|c|c|c|c|c|c|c|}
\hline 108 & 139.59 & 123.93 & 263.52 & 19.84 & 47.03 & 1.53 & 2.10 & 2.88 & $8.58 \mathrm{E}-27$ \\
\hline 109 & 140.89 & 125.65 & 266.54 & 19.89 & 47.14 & 1.53 & 2.11 & 2.90 & $8.54 \mathrm{E}-27$ \\
\hline 110 & 142.18 & 127.38 & 269.56 & 19.95 & 47.26 & 1.54 & 2.12 & 2.91 & $8.51 \mathrm{E}-27$ \\
\hline 111 & 143.47 & 129.13 & 272.60 & 20.01 & 47.37 & 1.54 & 2.13 & 2.93 & $8.48 \mathrm{E}-27$ \\
\hline 112 & 144.76 & 130.87 & 275.64 & 20.06 & 47.48 & 1.55 & 2.14 & 2.95 & $8.44 \mathrm{E}-27$ \\
\hline 113 & 146.06 & 132.63 & 278.69 & 20.12 & 47.59 & 1.55 & 2.14 & 2.96 & $8.41 \mathrm{E}-27$ \\
\hline 114 & 147.35 & 134.40 & 281.74 & 20.17 & 47.70 & 1.56 & 2.15 & 2.98 & $8.38 \mathrm{E}-27$ \\
\hline 115 & 148.64 & 136.17 & 284.81 & 20.23 & 47.81 & 1.56 & 2.16 & 3.00 & $8.35 \mathrm{E}-27$ \\
\hline 116 & 149.93 & 137.95 & 287.88 & 20.28 & 47.92 & 1.57 & 2.17 & 3.01 & $8.32 \mathrm{E}-27$ \\
\hline 117 & 151.23 & 139.74 & 290.96 & 20.33 & 48.03 & 1.57 & 2.18 & 3.03 & $8.29 \mathrm{E}-27$ \\
\hline 118 & 152.52 & 141.53 & 294.05 & 20.39 & 48.13 & 1.58 & 2.19 & 3.04 & $8.26 \mathrm{E}-27$ \\
\hline 119 & 153.81 & 143.33 & 297.15 & 20.44 & 48.24 & 1.58 & 2.20 & 3.06 & $8.23 \mathrm{E}-27$ \\
\hline 120 & 155.11 & 145.14 & 300.25 & 20.49 & 48.34 & 1.59 & 2.21 & 3.08 & $8.2 \mathrm{E}-27$ \\
\hline 121 & 156.40 & 146.96 & 303.36 & 20.55 & 48.44 & 1.59 & 2.22 & 3.09 & $8.17 \mathrm{E}-27$ \\
\hline 122 & 157.69 & 148.79 & 306.48 & 20.60 & 48.55 & 1.60 & 2.23 & 3.11 & $8.14 \mathrm{E}-27$ \\
\hline 123 & 158.98 & 150.62 & 309.60 & 20.65 & 48.65 & 1.60 & 2.24 & 3.12 & $8.11 \mathrm{E}-27$ \\
\hline 124 & 160.28 & 152.46 & 312.74 & 20.70 & 48.75 & 1.61 & 2.25 & 3.14 & $8.08 \mathrm{E}-27$ \\
\hline 125 & 161.57 & 154.31 & 315.88 & 20.76 & 48.85 & 1.61 & 2.26 & 3.15 & $8.05 \mathrm{E}-27$ \\
\hline 126 & 162.86 & 156.16 & 319.02 & 20.81 & 48.95 & 1.62 & 2.27 & 3.17 & $8.03 \mathrm{E}-27$ \\
\hline 127 & 164.15 & 158.03 & 322.18 & 20.86 & 49.05 & 1.62 & 2.27 & 3.19 & $8 \mathrm{E}-27$ \\
\hline 128 & 165.45 & 159.90 & 325.34 & 20.91 & 49.15 & 1.63 & 2.28 & 3.20 & 7.97E-27 \\
\hline 129 & 166.74 & 161.78 & 328.51 & 20.96 & 49.24 & 1.63 & 2.29 & 3.22 & $7.94 \mathrm{E}-27$ \\
\hline 130 & 168.03 & 163.66 & 331.69 & 21.01 & 49.34 & 1.64 & 2.30 & 3.23 & $7.92 \mathrm{E}-27$ \\
\hline 131 & 169.32 & 165.55 & 334.87 & 21.06 & 49.44 & 1.64 & 2.31 & 3.25 & $7.89 \mathrm{E}-27$ \\
\hline 132 & 170.62 & 167.45 & 338.07 & 21.11 & 49.53 & 1.65 & 2.32 & 3.26 & $7.87 \mathrm{E}-27$ \\
\hline 133 & 171.91 & 169.36 & 341.27 & 21.16 & 49.63 & 1.65 & 2.33 & 3.28 & $7.84 \mathrm{E}-27$ \\
\hline 134 & 173.20 & 171.27 & 344.47 & 21.21 & 49.72 & 1.66 & 2.34 & 3.29 & $7.81 \mathrm{E}-27$ \\
\hline 135 & 174.49 & 173.19 & 347.68 & 21.26 & 49.81 & 1.66 & 2.34 & 3.31 & $7.79 \mathrm{E}-27$ \\
\hline 136 & 175.79 & 175.12 & 350.91 & 21.31 & 49.91 & 1.67 & 2.35 & 3.32 & $7.76 \mathrm{E}-27$ \\
\hline 137 & 177.08 & 177.05 & 354.13 & 21.36 & 50.00 & 1.67 & 2.36 & 3.34 & $7.74 \mathrm{E}-27$ \\
\hline
\end{tabular}

\section{Discussion on the Nature of Dark Matter}

We would like to appeal that, when $95 \%$ of total cosmic mass is believed to be in the form of dark nature having interactions only with gravitation, it may not be logical to attribute its nature to any known or unknown elementary particle supposed to be originating from interactions involved with visible mass spectrum having negligible gravity at atomic, nuclear and electroweak scales.

As current cosmic temperature is at $2.7 \mathrm{~K}$, recently it has been suggested that, 
galactic cold hydrogen can be considered as dark matter [77] [78]. Since hydrogen is the basic building block of visible matter, this proposal can be given a chance. If so, $95 \%$ of the cosmic mass must be explained with a suitable mechanism with respect to current sub zero temperatures, past high temperatures and dark matter needed for the formation and evolution of galaxies. For example, based on our approach, estimated dark mass of current Milky Way is 24.5 times of its visible mass. It needs a reasonable mechanism for generating the required dark matter distribution.

If dark matter is really having a different nature and if one is willing to study and understand the mechanism of transformation of dark mass to Hydrogen atoms, it may give some clue. Applying our idea to Sun and Proton, their current dark masses are $1.5 \times 10^{26} \mathrm{~kg}$ and $3.6 \times 10^{-60} \mathrm{~kg}$ respectively. With these magnitudes, it is possible to say that, at atomic level, at present, dark matter influence is negligible. Even for Sun like massive stars, dark matter is having a very little role. This can be confirmed with current gravitational observations. We are working in this direction.

Somehow, by any theory or by any other means, if one is willing to fix the proposed dark mass reference, $\left(M_{X}\right) \cong 3.523 \times 10^{38} \mathrm{~kg}$ as a constant throughout the cosmic evolution [79], it may help in coupling it with elementary building blocks of particle physics. With reference to the estimated dark mass of proton, we noticed one very interesting relation for fitting the strong coupling constant, $\alpha_{s}$ [80]. It can be expressed in the following way.

$$
\left(\frac{1}{\alpha_{s}}\right) \cong \sqrt{\ln \left(\frac{m_{p}}{m_{\text {pdark }}}\right)} \cong \sqrt{\ln \left(\frac{1.67262 \times 10^{-27} \mathrm{~kg}}{3.6 \times 10^{-60} \mathrm{~kg}}\right)} \cong \frac{1}{0.1153}
$$

This value is closely matching with our recent estimation connected with three large atomic gravitational constants [81].

\section{To Check the Scope and Validity of Our Model}

Unless it explains the current observations and predict fresh ideas, no new model of cosmology can be encouraged for further investigation. In this context, we appeal the readers to go through the following points.

1) We clearly demonstrate the role of Planck scale in fitting the current cosmic Hubble parameter and this procedure is independent of galactic red shifts. It can certainly pave a way for developing a realistic model of "quantum cosmology" [21].

2) Based on the requirement of spin in quantum gravity, we tried to explain various applications of cosmic rotation in estimating cosmic radius, mass, temperature, Hubble parameter, galactic flat rotation speed, galactic radius and galactic angular velocity.

3) By considering whole cosmic mass as dark matter, we proposed a simple method for estimating galactic dark mass.

4) Big bang, Inflation, Nucleosynthesis, baryonic acoustic oscillations and 
current acceleration are the key pillars of standard cosmology [82]. In this context, we would like to appeal that,

a) As known physics is helpless in quantifying "Big bang" physical conditions, we propose a mechanism of "growing Planck ball". This logic seems to be simple and easy to implement.

b) As there is no proper theoretical reasoning for the origin of "inflation" [38], it's well believed end results like "flatness", "homogeneity" and "isotropy" can also be understood with continuous and prolonged light speed expansion. This logic is natural, non-controversial, simple to follow and simple to implement.

c) As an end result of inflation, standard cosmology is giving a top priority for exponential cosmic expansion in size, but no way considering its corresponding exponential increase in cosmic time. When it is strongly believed that, space-time is inseparable quantities, it seems illogical to believe in the standard model of narrow time line of $\left(1 / H_{0}\right)$ associated with post inflation.

d) According to standard model of cosmology, primordial nucleosynthesis seems to be happened in between first ( 3 to 20) minutes of big bang. Expected approximate cosmic temperature range for this characteristic time period is $\left(10^{9}\right.$ to $\left.10^{7}\right) \mathrm{K}$. Based on relations (1) to (20), Time-Temperature relation can be expressed as,

$$
T_{t} \cong\left(\frac{9 c^{2}}{20 \pi G a t^{2}}\right)^{\frac{1}{4}} \cong \frac{2.247 \times 10^{10}}{\sqrt{t}}
$$

where, $t=$ Time expressed in seconds.

$T_{t}=$ Cosmic temperature correspond to time $t$.

Based on this relation, estimated decreasing temperature range correspond to first ( 3 to 20 ) minutes is $\left(1.675 \times 10^{9}\right.$ to $\left.64.9 \times 10^{7}\right) \mathrm{K}$. In our model, by knowing the mass density or temperature required for generation of primordial nucleons and atoms, it seems possible to understand the complete nucleosynthesis in a natural manner with an increase in cosmic time line.

e) Current characteristic galactic separation distance of 500 Mly pertaining to Baryonic Acoustic Oscillations [83] [84] can be approximated with the following simple relation.

$$
\left(l_{\text {sep }}\right)_{0} \approx\left(\frac{\left[\left(V_{\text {Grot }}\right)_{0 A}+\left(V_{\text {Grot }}\right)_{0 B}\right] / 2}{c}\right) R_{0} \approx\left(\frac{\left(V_{\text {Grot }}\right)_{0 A}+\left(V_{\text {Grot }}\right)_{0 B}}{2 \omega_{0}}\right)
$$

where,

$R_{0} \cong \frac{C}{\omega_{0}}=$ Current cosmic radius.

$\left(V_{G r o t}\right)_{0 A}=$ Flat rotation speed of galaxy A.

$\left(V_{\text {Grot }}\right)_{0 B}=$ Flat rotation speed of galaxy $\mathrm{B}$.

$\left(l_{\text {sep }}\right)_{0}=$ Current separation distance of galaxy A and galaxy B.

For two Milky Way like galaxies, separation distance is around 1440 Mly and it is 2.8 times higher than $\mathrm{BAO}$ scale. It needs further study. In a scaling ap- 
proach,

$$
\begin{aligned}
&\left(l_{\text {sep }}\right)_{0} \propto\left(\frac{\left[\left(V_{\text {Grot }}\right)_{0 A}+\left(V_{\text {Grot }}\right)_{0 B}\right]}{2}\right) \\
&\left(l_{\text {sep }}\right)_{0} \propto R_{0}
\end{aligned}
$$

f) In section (16), we have proposed a reasonable explanation for understanding internal cosmic acceleration having light speed expansion at boundary.

g) In section (15), we have proposed a reasonable explanation for understanding cosmic anisotropy spreading towards cosmic boundary.

5) Considering Planck scale and current cosmic temperatures, it seems possible to understand the issues connected with currently believed cosmological constant problem in terms of the ratio of Planck scale thermal energy density and current thermal energy density.

6) By estimating the average mass of a galaxy with reference to average flat rotation speed (of so many galaxies) and by considering the current cosmic mass and volume, number of galaxies and their average distance can be estimated easily. Its approximate relation can be expressed as follows.

$$
l_{\text {ave }} \approx\left(\frac{4 \pi R_{0}^{3}}{3\left(M_{0} / M_{x}\right)}\right)^{\frac{1}{3}} \approx\left(1.3 \times 10^{30} M_{x}\right)^{\frac{1}{3}}
$$

where, $l_{\text {ave }}=$ Average galactic distance,

$M_{x}=$ Average galactic mass,

$M_{0}=$ Current cosmic mass,

$R_{0}=$ Current cosmic radius,

$\left(M_{0} / M_{x}\right)=$ Approximate number of galaxies.

7) Based on relations (11) and (50), at any stage of cosmic evolution, ratio of cosmic thermal energy and energy of graviton is close to 0.6831 .

8) With further study, it seems possible to understand the factors helping photon or graviton to move at "light speed" can be understood. Proceeding further, factors helping the universe to move at "light speed" can also be understood and thereby, issues connected with "dark energy" can be resolved.

9) By considering whole cosmic mass as dark matter, it seems possible to understand the issues connected with "nature" of dark matter.

\section{Conclusions}

Considering the points and relations proposed in sections (2) to (27), our model can be recommended for further research. We would like to emphasize the point that, "space" and "matter" are inseparable cosmic entities and like matter, space cannot travel faster than speed of light.

Flatness problem can be understood well with Machian radius of the current universe. Considering light speed expansion, inflation and dark energy concepts can be relinquished. Based on relations (4) and (8), Hubble parameter can be es- 
timated independent of galactic distances and their red shifts.

Even though, cosmic horizon is assumed to be expanding at light speed, based on relations (47) and (52), it seems possible to have internal acceleration below the cosmic horizon and seems to be a consequence of Hubble's law for increasing time periods. Even though, estimated cosmic radius is 146.3 times of the Hubble radius, estimated angular velocity is 146.3 times less than the Hubble parameter and is directly helping in estimating galactic masses and working radii with relations (21) to (41).

Further study and advanced telescopes may help in thoroughly exploring the cosmic and galactic structures in a broad view based on the concepts of Quantum Cosmology. In this context, relation (8) can be given some consideration.

\section{Acknowledgements}

Author Seshavatharam is grateful to Dr. E.T. Tatum for guiding with his valuable scientific thoughts on "light speed expansion" and "Flat Space Cosmology". Author Seshavatharam is indebted to Professors Shri M. Nagaphani Sarma, Chairman, Shri K.V. Krishna Murthy, founder Chairman, Institute of Scientific Research in Vedas (I-SERVE), Hyderabad, India and Shri K.V.R.S. Murthy, former scientist IICT (CSIR), Govt. of India, Director, Research and Development, I-SERVE, for their valuable guidance and great support in developing this subject. Both the authors are thankful to the anonymous reviewers for their helpful suggestions in improving the quality of the paper.

\section{Conflicts of Interest}

The authors declare no conflicts of interest regarding the publication of this paper.

\section{References}

[1] Friedmann, A. (1999) On the Curvature of Space. General Relativity and Gravitation, 31, 1991-2000. https://doi.org/10.1023/A:1026751225741

[2] Hawking, S.W. (1988) A Brief History of Time. Bantam Dell Publishing Group, New York.

[3] Seshavatharam, U.V.S. (2010) Physics of Rotating and Expanding Black Hole Universe. Progress in Physics, 4, 7-14.

[4] Seshavatharam, U.V.S. (2012) The Primordial Cosmic Black Hole and the Cosmic Axis of Evil. International Journal of Astronomy, 1, 20-37. https://doi.org/10.1155/2012/805402

[5] Seshavatharam, U.V.S. and Lakshminarayana, S. (2013) Applications of Hubble Volume in Atomic Physics, Nuclear Physics, Particle Physics, Quantum Physics and Cosmic Physics. Journal of Nuclear Physics, Material Sciences, Radiation and Applications, 1, 45-60. https://doi.org/10.15415/jnp.2013.11005

[6] Seshavatharam, U.V.S. and Lakshminarayana, S. (2014) Friedman Cosmology: Reconsideration and New Results. International Journal of Astronomy, Astrophysics and Space Science, 1, 16-26. 
[7] Seshavatharam, U.V.S. and Lakshminarayana, S. (2014) On the Evolving Black Holes and Black Hole Cosmology-Scale Independent Quantum Gravity Approach. Frontiers of Astronomy, Astrophysics and Cosmology, 1, 1-15.

[8] Seshavatharam, U.V.S. and Lakshminarayana, S. (2015) Black Hole Cosmology with Propelling Lambda Term \& Hindu Cosmic Age. Prespacetime Journal, 6, 850-874.

[9] Seshavatharam, U.V.S. and Lakshminarayana, S. (2015) Role of Mach's Principle and Quantum Gravity in Understanding Cosmic Evolution and Cosmic Red-Shift. Frontiers of Astronomy, Astrophysics and Cosmology, 1, 24-30.

[10] Seshavatharam, U.V.S., Tatum, E.T. and Lakshminarayana, S. (2015) On the Role of Gravitational Self Energy Density in Spherical Flat Space Quantum Cosmology. Journal of Applied Physical Science International, 4, 228-236.

[11] Seshavatharam, U.V.S. and Lakshminarayana, S. (2015) Applications of Light Speed Expansion and Gravitational Self-Energy Density in Black Hole Cosmology. International Journal of Advanced Astronomy, 3, 123-128. https://doi.org/10.14419/ijaa.v3i2.5418

[12] Tatum, E.T., Seshavatharam, U.V.S. and Lakshminarayana, S. (2015) The Basics of Flat Space Cosmology. International Journal of Astronomy and Astrophysics, 5, 116-124. https://doi.org/10.4236/ijaa.2015.52015

[13] Seshavatharam, U.V.S. and Lakshminarayana, S. (2015) Primordial Hot Evolving Black Holes and the Evolved Primordial Cold Black Hole Universe. Frontiers of Astronomy, Astrophysics and Cosmology, 1, 16-23.

[14] Seshavatharam, U.V.S. and Lakshminarayana, S. (2016) Cosmologically Strengthening Hydrogen Atom in Black Hole Universe. Journal of Nuclear Physics, Material Sciences, Radiation and Applications, 3, 265-278. https://doi.org/10.15415/jnp.2016.32024

[15] Seshavatharam, U.V.S. and Lakshminarayana, S. (2016) On the Possible Role of Continuous Light Speed Expansion in Black Hole \& Gravastar Cosmology. Prespacetime Journal, 7, 584-600.

[16] Seshavatharam, U.V.S. and Lakshminarayana S. (2018) Toy Model of Evolving and Spinning Quantum Cosmology-A Review. International Journal of Astronomy, Astrophysics and Space Science, 5, 1-13.

[17] Seshavatharam, U.V.S. and Lakshminarayana, S. (2019) A First Step in Evolving Quantum Cosmology. Journal of Physics. Conf. Series, 1251, Article ID: 012045. https://doi.org/10.1088/1742-6596/1251/1/012045

[18] Seshavatharam, U.V.S. and Lakshminarayana, S. (2019) An Outline Picture of a Growing and Rotating Planck Universe with Emerging Dark Foam. Asian Journal of Research and Reviews in Physics, 2, 1-13. https://doi.org/10.9734/ajr2p/2019/v2i230097

[19] Seshavatharam, U.V.S. and Lakshminarayana, S. (2019) A Practical Model of Godel-Planck-Hubble-Birch Universe. Athens Journal of Sciences, 6, 211-230. https://doi.org/10.30958/ajs.6-3-4

[20] Seshavatharam, U.V.S. and Lakshminarayana, S. (2020) Light Speed Expansion and Rotation of a Primordial Cosmic Black Hole Universe Having Internal Acceleration. International Astronomy and Astrophysics Research Journal, 2, 9-27. https://journaliaarj.com/index.php/IAARJ/article/view/18/25

[21] Bojowald, M. (2015) Quantum Cosmology: A Review. Reports on Progress in Physics, 78, Article ID: 023901. https://doi.org/10.1088/0034-4885/78/2/023901

[22] Nielsen, J.T., Guffanti, A. and Sarkar, S. (2016) Marginal Evidence for Cosmic Ac- 
celeration from Type Ia Supernovae. Scientific Reports, 6, Article No. 35596. https://doi.org/10.1038/srep35596

[23] Wei, J.J., Wu, X.F., Melia, F. and Maier, R.S. (2015) Comparative Analysis of the Supernova Legacy Survey Sample with $\Lambda \mathrm{CDM}$ and the $R_{\mathrm{h}}=c t$ Universe. The Astronomical Journal, 149, 102. https://doi.org/10.1088/0004-6256/149/3/102

[24] Melia, F. and Fatuzzo, M. (2016) The Epoch of Reionization in the $R_{\mathrm{h}}=c t$ Universe. Monthly Notices of Royal Astronomical Society, 456, 3422-3431. https://doi.org/10.1093/mnras/stv2902

[25] Sapar, A. (2019) A Perpetual Mass-Generating Planckian Universe. Proceedings of the Estonian Academy of Sciences, 68, 1-12. https://doi.org/10.3176/proc.2019.1.01

[26] Netchitailo, V.S. (2020) World-Universe Model-Alternative to Big Bang Model. Journal of High Energy Physics, Gravitation and Cosmology, 6, 133-158. https://doi.org/10.4236/jhepgc.2020.61012

[27] Whitrow, G.J. (1946) The Mass of the Universe. Nature, 158, 165-166. https://doi.org/10.1038/158165b0

[28] Sciama, D.W. (1953) On the Origin of Inertia. Monthly Notices of the Royal Astronomical Society, 113, 34-42. https://doi.org/10.1093/mnras/113.1.34

[29] Dicke, R.H. (1959) Gravitation-An Enigma. American Scientist, 47, 25-40.

[30] Dicke, R.H. (1959) New Research on Old Gravitation: Are the Observed Physical Constants Independent of the Position, Epoch, and Velocity of the Laboratory? Science, 129, 621-624. https://doi.org/10.1126/science.129.3349.621

[31] Brans, C. and Dicke, R.H. (1961) Mach's Principle and a Relativistic Principle of Gravitation. Physical Review, 124, 925-935.

https://doi.org/10.1103/PhysRev.124.925

[32] Arbab, A.I. (2004) Quantization of Gravitational System and Its Cosmological Consequences. General Relativity and Gravitation, 36, 2465-2479. https://doi.org/10.1023/B:GERG.0000046833.08113.6b

[33] Arbab, A.I. (2001) Large Scale Quantization and the Essence of the Cosmological Problems. Spacetime \& Substance, 2, 55.

[34] Christillin, P. and Barattini, L. (2012) The Machian Contribution of the Universe to Geodetic Precession, Frame Dragging and Gravitational Clock Effect. arXiv: 1206.4593v2.

[35] Barattini, L. and Christillin, P. (2012) The Machian Origin of the Centrifugal Force. Journal of Modern Physics, 3, 1298-1300. https://doi.org/10.4236/jmp.2012.329167

[36] Abbott, B.P., et al. (2016) Observation of Gravitational Waves from a Binary Black Hole Merger. Physical Review Letters, 116, Article ID: 061102. https://doi.org/10.1103/PhysRevLett.116.061102

[37] Abhas, M. (2011) Why the Big Bang Model Cannot Describe the Observed Universe Having Pressure and Radiation. Journal of Modern Physics, 2, 1436-1442. https://doi.org/10.4236/jmp.2011.212177

[38] Ljjas, A., Steinhardt, P.J. and Abraham, L. (2014) Inflationary Schism. Physics Letters B, 736, 142-146. https://doi.org/10.1016/j.physletb.2014.07.012

[39] Ade, P.A.R., et al. (2016) Planck Collaboration: Planck 2015 Results. XIII. Cosmological Parameters. Astronomy \& Astrophysics, 594, Article No. A13.

[40] Korotky, V.A., Masár, E. and Obukhov, Y.N. (2020) In the Quest for Cosmic Rotation. Universe, 6, 14. https://doi.org/10.3390/universe6010014

[41] Mcgaugh, S. (2020) Predictions and Outcomes for the Dynamics of Rotating Galax- 
ies. Galaxies, 8, 35. https://doi.org/10.3390/galaxies8020035

[42] Godel, K. (1950) Rotating Universes in General Relativity Theory. Proceedings of the International Congress of Mathematicians in Cambridge, 1, 175-181.

[43] Birch, P. (1982) Is the Universe Rotating? Nature, 298, 451-454. https://doi.org/10.1038/298451a0

[44] Obukhov, Y.N. (2000) On Physical Foundations and Observational Effects of Cosmic Rotation Colloquium on Cosmic Rotation. In: Scherfner, M., Chrobok, T. and Shefaat, M., Eds., Colloquium on Cosmic Rotation, Wissenschaft Und Technik Verlag, Berlin, 23-96.

[45] Godlowski, W. (2011) Global and Local Effects of Rotation: Observational Aspects. International Journal of Modern Physics, 20, 1643-1673. https://doi.org/10.1142/S0218271811019475

[46] Longo, M.J. (2011) Detection of a Dipole in the Handedness of Spiral Galaxies with Redshifts z 0.04. Physics Letters B, 699, 224-229.

https://doi.org/10.1016/j.physletb.2011.04.008

[47] Chechin, L.M. (2017) Does the Cosmological Principle Exist in the Rotating Universe? Gravitation and Cosmology, 23, 305-310.

[48] Whittaker, E.T. (1945) Spin in the Universe. Yearbook of Royal Society. Edinburgh: 513.

[49] Valery, T. and Timkov, S.V. (2015) Rotating Space of the Universe as a Source of Dark Energy and Dark Matter. The International Scientific-Technical Magazine: Measuring and Computing Devices in Technological Processes. Khmelnitsky National University, Khmelnitsky, Ukraine, 3, 200-204.

[50] Shamir, L. (2020) Multipole Alignment in the Large-Scale Distribution of Spin Direction of Spiral Galaxies. arXiv: 2004.02963v3.

[51] Clowe, D., et al. (2006) A Direct Empirical Proof of the Existence of Dark Matter. The Astrophysical Journal Letters, 648, L109-L113. https://doi.org/10.1086/508162

[52] Arun, K., Gudennavar, S.B. and Sivaram, C. (2017) Dark Matter, Dark Energy, and Alternate Models: A Review. Advances in Space Research, 60, 166-186. https://doi.org/10.1016/j.asr.2017.03.043

[53] Christillin, P. (2016) Cosmogonic Speculations: Particle Creation from Energy Conservation in the Universe Evolution. Journal of Modern Physics, 7, 1331-1344. https://doi.org/10.4236/jmp.2016.711119

[54] Brownstein J. R. and Moffat J. W. (2006) Galaxy Rotation Curves Without Non-Baryonic Dark Matter. The Astrophysical Journal, 636, 721-741. https://doi.org/10.1086/498208

[55] Milgrom, M. (1983) A Modification of the Newtonian Dynamics as a Possible Alternative to the Hidden Mass Hypothesis. The Astrophysical Journal, 270, 365-370. https://doi.org/10.1086/161130

[56] Sanders, R.H. (1998) Cosmology with Modified Newtonian Dynamics (MOND). Monthly Notices of the Royal Astronomical Society, 296, 1009-1018. https://doi.org/10.1046/j.1365-8711.1998.01459.x

[57] Courteau, S., et al (2014) Galaxy Masses: A Review. Reviews of Modern Physic, 86, 47-119. https://doi.org/10.1103/RevModPhys.86.47

[58] Narlikar, J.V. (2002) An Introduction to Cosmology. 3rd Edition, Cambridge University Press, Cambridge.

[59] Hubble, E.P. (1929) A Relation between Distance and Radial Velocity among Ex- 
tra-Galactic Nebulae. Proceedings of the National Academy of Sciences of the United States of America, 15, 168-173. https://doi.org/10.1073/pnas.15.3.168

[60] Giovannini, M. (2020) Primordial Backgrounds of Relic Gravitons. Progress in Particle and Nuclear Physics, 112, Article ID: 103774.

https://doi.org/10.1016/j.ppnp.2020.103774

[61] Christillin, P. (2014) The Machian Origin of Linear Inertial Forces from Our Gravitationally Radiating Black Hole Universe. The European Physical Journal Plus, 129, Article No. 175. https://doi.org/10.1140/epjp/i2014-14175-2

[62] Aluri, P.K. and Jain, P. (2012) Large Scale Anisotropy Due to Pre-Inflationary Phase of Cosmic Evolution. Modern. Physical Letters A, 27, Article ID: 1250014. https://doi.org/10.1142/S0217732312500149

[63] Perlmutter, S., et al. (1999) Measurements of $\Omega$ and $\Lambda$ from 42 High-Redshift Supernovae. The Astrophysical Journal, 517, 565-586. https://doi.org/10.1086/307221

[64] Riess, A.G., et al. (1998) Observational Evidence from Supernovae for an Accelerating Universe and a Cosmological Constant. The Astronomical Journal, 116, 1009-1038. https://doi.org/10.1086/300499

[65] Wang, D. and Meng, X.H. (2017) No Evidence for Dynamical Dark Energy in Two Models. Physical Review D, 96, Article ID: 103516. https://doi.org/10.1103/PhysRevD.96.103516

[66] Dam, L.H., et al. (2017) Apparent Cosmic Acceleration from Type Ia Supernovae. Monthly Notices of the Royal Astronomical Society, 472, 835-851. https://doi.org/10.1093/mnras/stx1858

[67] Gamow, G. (1948) The Origin of Elements and the Separation of Galaxies. Physical Review, 74, 505-506. https://doi.org/10.1103/PhysRev.74.505.2

[68] Hawking, S.W. (1975) Particle Creation by Black Holes. Communications in Mathematical Physics, 43, 199-220. https://doi.org/10.1007/BF02345020

[69] Watkins, L.L., et al. (2019) Evidence for an Intermediate-Mass Milky Way from Gaia DR2 Halo Globular Cluster Motions. The Astrophysical Journal, 873, 118. https://doi.org/10.3847/1538-4357/ab089f

[70] Deason, A.J., et al. (2020) The Edge of the Galaxy. Monthly Notices of the Royal Astronomical Society, 496, 3929-3942. https://arxiv.org/abs/2002.09497v3

[71] Bhattacharjee, P., et al. (2014) Rotation Curve of the Milky Way out to 200 Kpc. The Astrophysical Journal, 785, 63. https://doi.org/10.1088/0004-637X/785/1/63

[72] Sofue, Y. (2013) Rotation Curve and Mass Distribution in the Galactic Centre-From Black Hole to Entire Galaxy. Publications of the Astronomical Society of Japan, 65, 118. https://doi.org/10.1093/pasj/65.6.118

[73] Sofue, Y. (2020) Rotation Curve of the Milky Way and the Dark Matter Density. Galaxies, 8, 37. https://doi.org/10.3390/galaxies8020037

[74] Ogle, P.M., et al. (2019) A Break in Spiral Galaxy Scaling Relations at the Upper Limit of Galaxy Mass. The Astrophysical Journal Letters, 884, L11. https://doi.org/10.3847/2041-8213/ab459e

[75] Kirby, E.N., et al. (2013) Segue 2: The Least Massive Galaxy. The Astrophysical Journal, 770, 16. https://doi.org/10.1088/0004-637X/770/1/16

[76] Lelli, F., Mcgaugh, S.S. and Schombert, J.M. (2016) SPARC: Mass Models for 175 Disk Galaxies with Spitzer Photometry and Accurate Rotation Curves. The Astronomical Journal, 152, 157. https://doi.org/10.3847/0004-6256/152/6/157 
[77] Tatum, E.T. (2020) Dark Matter as Cold Atomic Hydrogen in Its Lower Ground State. Book Chapter.

[78] Holmlid, L. (2019) Ultra-Dense Hydrogen H(o) as Dark Matter in the Universe: New Possibilities for the Cosmological Red-Shift and the Cosmic Microwave Background Radiation. Astrophysics and Space Science, 364, Article No. 141. https://doi.org/10.1007/s10509-019-3632-y

[79] Seshavatharam, U.V.S. and Lakshminarayana, S. (2020) To Correlate Galactic Dark 28 and Visible Masses and to Fit Flat Rotation Speeds via MOND Approach and Cos29 mic Angular Acceleration. International Astronomy and Astrophysics Research Journal, 2, 28-43.

https://www.journaliaarj.com/index.php/IAARJ/article/view/24

[80] Tanabashi, M., et al. (2018) Particle Data Group. Physical Review D, 98, Article ID: 030001.

[81] Seshavatharam, U.V.S. and Lakshminarayana, S. (2019) On the Role of Four Gravitational Constants in Nuclear Structure. Mapana Journal of Sciences, 18, 21-45. https://doi.org/10.12723/mjs.48.2

[82] López-Corredoira, M. (2017) Tests and Problems of the Standard Model in Cosmology. Foundations of Physics, 47, 711-768. https://doi.org/10.1007/s10701-017-0073-8

[83] Eisenstein, D.J., et al. (2005) Detection of the Baryon Acoustic Peak in the Large-Scale Correlation Function of SDSS Luminous Red Galaxies. The Astrophysical Journal, 633, 560-574. https://doi.org/10.1086/466512

[84] Hernández-Aguayo, C., Cautun, M., Smith, A., Baugh, C.M. and Li, B.J. (2020) Measuring the Baryon Acoustic Oscillation Peak Position with Different Galaxy Selections. Monthly Notices of the Royal Astronomical Society, 494, 3120-3130. https://doi.org/10.1093/mnras/staa973 